Bull. Soc. math. France

130 (2), 2002, p. 169-209

\title{
PROBLÈME DE PLATEAU COMPLEXE DANS LES VARIÉTÉS KÄHLÉRIENNES
}

\author{
PAR FRÉDÉRIC SARKIS
}

\begin{abstract}
RÉSumÉ. - L'étude du «problème de Plateau complexe » (ou «problème du bord ») dans une variété complexe $X$ consiste à caractériser les sous-variétés réelles $\Gamma$ de $X$ qui sont le bord de sous-ensembles analytiques de $X \backslash \Gamma$. Notre principal résultat traite le cas $X=U \times \omega$ où $U$ est une variété complexe connexe et $\omega$ est une variété kählérienne disque convexe. Comme conséquence, nous obtenons des résultats de HarveyLawson [19], Dolbeault-Henkin [12] et Dinh [10]. Nous obtenons aussi une généralisation des théorèmes de Hartogs-Levi et Hartogs-Bochner. Finalement, nous montrons qu'une structure $\mathrm{CR}$ strictement pseudo-convexe plongeable dans une variété kählérienne disque-convexe est plongeable dans $\mathbb{C}^{n}$ si et seulement si elle admet une fonction CR non constante.

Abstract (Complex Plateau problem in Kähler manifolds). — The "complex Plateau problem" (or "boundary problem") in a complex manifold $X$ is the problem of characterizing the real submanifolds $\Gamma$ of $X$ which are boundaries of analytic subvarieties of $X \backslash \Gamma$. Our principal result treats the case $X=U \times \omega$ where $U$ is a connected complex manifold and $\omega$ is a disk-convex Kähler manifold. As a consequence, we obtain results of Harvey-Lawson [19], Dolbeault-Henkin [12] and Dinh [10]. We also give a generalization of Hartogs-Levi and Hartogs-Bochner theorems. Finally, we prove that a strictly pseudoconvex $\mathrm{CR}$ structure embeddable in a disk-convex Kähler manifold is embeddable in $\mathbb{C}^{n}$ if and only if it has a non constant CR function.
\end{abstract}

Texte reçu le 23 septembre 1999, révisé le 28 novembre 2000, accepté le 4 mai 2001 FRÉDÉRIC SARKIS, Laboratoire de Géométrie, Analyse, Topologie, Université de Lille I, USTL, 59655 Villeneuve d'Ascq Cedex (France) • E-mail : sarkis@agat.univ-lille1.fr Classification mathématique par sujets (2000). - 32F25, 32F40, 32D15, 32C30.

Mots clefs. - Problème du bord, problème de Plateau complexe, variété kählérienne, extension du type Hartogs, plongement CR, structure CR. 


\section{Introduction}

L'étude du «problème de Plateau complexe » (ou «problème du bord ») dans une variété complexe $X$ consiste à caractériser les sous-variétés réelles $\Gamma$ de $X$ qui sont le bord (au sens des courants) de sous-ensembles analytiques de $X \backslash \Gamma$.

Dans l'espace affine, les courbes bords de surfaces de Riemann sont caractérisées par une condition intégrale nommée « condition des moments » (voir [39], [37], [1], [28], [8]). Les sous-variétés réelles compactes, fermées et de dimension supérieure ou égale à trois qui sont bords d'ensembles analytiques de $\mathbb{C}^{n}$ sont celles dont la dimension de l'espace complexe tangent est maximale en chaque point (voir [18], [7], [8]).

Dans l'espace projectif, sous la condition précédente, le problème du bord n'admet pas toujours de solution. Harvey et Lawson [19] ont cependant donné une caractérisation en terme de condition des moments pour le problème du bord dans $P_{n}(\mathbb{C}) \backslash P_{n-p}(\mathbb{C})$ (où $2 p-1$ (avec $p \geq 2$ ) est la dimension de la variété considérée).

Récemment, Dolbeault et Henkin [12] (puis Dinh [8], [10]) ont donné une condition nécessaire et suffisante : le problème du bord pour une variété maximalement complexe $M \subset P_{n}(\mathbb{C})$ admet une solution s'il en admet une pour un nombre «assez grand» de tranches de $M$ par des sous-espaces linéaires.

Le but de cet article est de généraliser ce dernier résultat à une variété produit $U \times \omega$ où $U$ est une variété complexe connexe et $\omega$ est une variété kählérienne compacte (ou plus généralement disque convexe). L'étude du problème du bord dans les espaces produits n'est pas restrictive. En effet, le problème du bord dans l'espace projectif peut toujours être réduit à l'étude du problème du bord dans un espace produit. Comme nous le verrons, la réciproque n'est en général pas vraie. De plus, cette résolution parait la plus adaptée pour l'étude de l'extension des applications CR car si $f$ est une application CR, le graphe de $f$ est naturellement dans un espace produit. Soit $X$ une variété kählérienne disque convexe; nous obtenons alors les corollaires suivants :

1) Une nouvelle démonstration de la caractérisation géométrique du problème du bord dans $P_{n}(\mathbb{C})$ donnée dans [12], [10].

2) Une généralisation du théorème de Hartogs-Levi pour les applications méromorphes à valeurs dans $X$. En particulier, nous retrouvons les généralisations données dans [10] et [22].

3) Une généralisation du théorème de Hartogs-Bochner pour les applications $\mathrm{CR}$ à valeurs dans une variété kählérienne disque convexe $X$ (pour $X=$ $P_{n}(\mathbb{C})$, nous retrouvons des résultats de [12], [30], [33]).

4) La généralisation suivante du théorème de Hartogs-Bochner : supposons que $X$ est une variété kählérienne de dimension 2 ne contenant aucune surface de Riemann compacte. Soit $M$ une hypersurface réelle compacte et connexe de $X$ la séparant en deux composantes connexes $\Omega_{1}$ et $\Omega_{2}$. Alors toute fonction

TOME $130-2002-\mathrm{N}^{\mathrm{O}} 2$ 
holomorphe au voisinage de $M$ admet une extension holomorphe sur $\Omega_{1}$ ou sur $\Omega_{2}$. De plus, on peut choisir l'ouvert indépendant de la fonction.

Ainsi que le corollaire principal suivant nouveau même dans le cas où $X=P_{n}(\mathbb{C}):$

5) Soit $M$ une structure $\mathrm{CR}$ abstraite, strictement pseudoconvexe de dimension $2 p-1(p \geq 2)$ et plongeable dans $X$. La variété $M$ est plongeable dans $\mathbb{C}^{n}$ (ou de manière équivalente, admet une solution au problème du bord dans $X$ ) si et seulement si elle admet une fonction CR non constante.

Ce dernier résultat donne une nouvelle réduction du problème suivant (voir [12]) : Soit $M$ une variété strictement pseudoconvexe de $P_{n}(\mathbb{C})$ de dimension $2 p-1(p \geq 2)$. La variété $M$ est-elle toujours le bord d'une p-chaîne holomorphe (ou de manière équivalente $M$ est elle plongeable dans l'espace affine) ? En particulier, cela prouve de manière immédiate que les exemples de structures CR d'Andreotti-Rossi [31], [32], [3], [16], [13] et de Barrett [4] (cas où l'espace des fonctions $\mathrm{CR}$ est de dimension 1) ne sont plongeables dans aucune variété kählérienne disque convexe $X$. En effet, ces variétés ne sont pas plongeables dans l'espace affine mais, par construction, elles admettent des fonctions $\mathrm{CR}$ non constantes, on en déduit alors directement qu'elles ne sont pas plongeables dans $X$ (pour $X=P_{n}(\mathbb{C})$ et $M$ la structure CR d'Andreotti-Rossi, ceci est démontré de manière différente dans [33]).

Dans toute la suite, les variétés considérées sont supposées dénombrables à l'infini et munies d'une métrique complète. Par volume p-dimensionnel, nous entendrons la mesure de Hausdorff de dimension $p$ associée à cette métrique. Nous nous intéresserons à montrer que certains compacts sont de volume $p$-dimensionnel fini ou nul. Or ces deux notions ne dépendent pas de la métrique choisie. Par conséquent tous les résultats que nous obtenons sont indépendants de la métrique fixée au départ.

Je voudrais remercier T.C. Dinh pour ses remarques sur cet article.

\section{Problème du bord dans les variétés produit : cas lisse}

2.1. Théorème principal. — Dans le même esprit que [23], nous définissons :

DÉFInItion 2.1. - Nous dirons qu'une variété complexe $\omega$ est disque convexe si pour tout compact $K \subset \omega$, il existe un compact $\widehat{K} \subset \omega$ tel que tout ensemble analytique $A$ irréductible et de dimension 1 de $\omega \backslash K$ tel que $A \cup K$ est un compact de $\omega$ et $\bar{A} \cap K \neq \varnothing$ vérifie $A \subset \widehat{K}$.

Bien sûr, toute variété compacte ou holomorphiquement convexe est disque convexe.

BULletin DE LA SOCIÉtÉ MATHÉMATIQUE DE FRANCE 
Nous rappelons qu'une sous-variété lisse $\Gamma$ de dimension $2 n-1$ d'une variété complexe $X$ est maximalement complexe si pour tout point $x \in \Gamma$, l'espace holomorphe tangent $H_{x}(\Gamma)=T_{x}(\Gamma) \cap J T_{x}(\Gamma)$ est de dimension complexe $n-1$ (où $T_{x}(\Gamma)$ est l'espace réel tangent à $\Gamma$ au point $x$ et $J$ est la multiplication complexe).

DÉfinition 2.2. - Soit $U$ une variété complexe. Un sous-ensemble $K \subset U$ sera dit $(n-1)$-générique s'il n'est pas inclus dans une réunion dénombrable d'ensembles analytiques de dimension $n-1$ immergés dans $U$.

- Soit $X$ un espace complexe (muni d'une métrique). On appelle p-chaîne holomorphe de $X$ toute somme localement finie

$$
[T]=\sum n_{j}\left[V_{j}\right]
$$

à coefficients $n_{j}$ dans $\mathbb{Z}$ de sous-ensembles analytiques $V_{j}$, deux à deux différents, de dimension $p$ et fermés de $X$.

- On appelle volume de [T], l'expression

$$
\operatorname{Vol}[T]=\sum\left|n_{j}\right| \operatorname{Vol} V_{j}
$$

où $\operatorname{Vol} V_{j}$ est le volume $2 p$-dimensionnel de l'ensemble analytique $V_{j} ; \operatorname{Vol}[T]$ est aussi la masse du courant $[T]$. On notera $T$ le support de la $p$-chaîne holomorphe $[T]$ (i.e. $\left.T=\bigcup_{\left\{j ; n_{j} \neq 0\right\}} V_{j}\right)$. Dans la suite, si $[T]$ est un courant, on notera $\operatorname{Vol}[T]$ la masse de ce courant et $T$ son support.

Soient $M$ et $N$ des variétés réelles lisses orientées et $f: M \rightarrow N$ une application lisse. Alors, d'après le théorème de Sard, pour presque tout $y \in N$, la tranche $f^{-1}(y)$ est soit vide soit une sous-variété lisse orientée de $M$ de codimension $\operatorname{dim} N$. Nous noterons $[M, f, y]$ le courant d'intégration sur cette tranche qui est alors bien défini.

ThÉORÈme 2.3. - Soient U une variété complexe connexe de dimension complexe $n$ et $\omega$ une variété kählérienne disque convexe. Soit $\Gamma$ une sous-variété lisse maximalement complexe de $U \times \omega$ et de dimension réelle $2 n+1$. Notons $\pi: U \times \omega \rightarrow U$ la projection canonique sur le premier membre. Supposons de plus que $\Gamma$ vérifie les propriétés suivantes:

(a) Pour tout compact $K \subset U, \Gamma \cap \pi^{-1}(K)$ est un compact de $U \times \omega$.

(b) Pour tout $z \in U$, il existe au plus un nombre fini de points $x$ de $\gamma_{z}=$ $\Gamma \cap\{z\} \times \omega$ tels que $\Gamma$ ne soit pas transverse $\grave{a}\{z\} \times \omega$ au point $x$.

(c) Pour tout $z \in U, \gamma_{z}$ est une courbe lisse par morceaux dont la partie régulière admet un nombre fini de composantes connexes.

Pour tout $z \in U$, le courant d'intégration sur $\gamma_{z}$ est alors bien défini et est noté $\left[\gamma_{z}\right]$. Alors les propositions suivantes sont équivalentes :

TOME $130-2002-\mathrm{N}^{\mathrm{O}} 2$ 
1) Il existe un sous-ensemble $(n-1)$-générique $Z$ de $U$ tel que pour tout $z \in Z$, il existe une 1-chaîne holomorphe $\left[S_{z}\right]$ de $(\{z\} \times \omega) \backslash \gamma_{z}$ de masse finie, dont l'adhérence $\bar{S}_{z}$ est un compact de $\{z\} \times \omega$ et telle que $d\left[S_{z}\right]=\left[\gamma_{z}\right]$ (au sens des courants).

2) Il existe un ouvert non vide $O \subset U$ tel que $[\Gamma]$ admette une solution au problème $d u$ bord dans $O \times \omega$ (i.e. il existe une $(n+1)$-chaîne holomorphe $[T]$ de $(O \times \omega) \backslash \Gamma$, de masse localement finie dans $O \times \omega$, telle que pour tout compact $K \subset O, \bar{T} \cap \pi^{-1}(K)$ est un compact de $O \times \omega$ et telle que $d[T]=[\Gamma]$ au sens des courants).

3) Il existe un ensemble $F \subset U$ de mesure de Hausdorff $(2 n-1)$-dimensionnelle nulle de sorte que pour tout point $z \in U \backslash F$, il existe un ouvert $O_{z} \subset U$ tel que $[\Gamma]$ admette une solution au problème du bord dans $O_{z} \times \omega$.

4) Il existe un fermé $Y \subset U$ de mesure de Hausdorff $2 n$-dimensionnelle nulle tel que $[\Gamma]$ admette une solution au problème du bord dans $(U \backslash Y) \times \omega$.

Par définition de courbes lisses par morceaux, les hypothèses du théorème impliquent que les courbes $\gamma_{z}$ considérées sont de longeur finie.

Contrairement à ce qui se passe dans le cas des variétés de Stein, il n'existe pas généralement de solution globale au problème du bord dans $U \times \omega$ comme le montre l'exemple suivant :

EXEMPLE 1. - Soient

$$
C(1,3)=\{z \in \mathbb{C} ; 1<|z|<3\}, \quad C(2)=\{z \in \mathbb{C} ;|z|=2\}
$$

et $\phi: \mathbb{C} \rightarrow \mathbb{C}^{2}$ définie par $\phi(z)=\left(z, \mathrm{e}^{1 / z}\right)$. Soient

$$
S=\phi(C(1,3)), \quad \gamma=\phi(C(2)) \quad \text { et } \quad \Gamma=\gamma \times P_{1}(\mathbb{C}) .
$$

Soit $\widetilde{\Gamma}$ une petite déformation de $\Gamma$ dans $S \times P_{1}(\mathbb{C})$ obtenue grâce à la proposition 2.4. Puisque $\widetilde{\Gamma}$ est une hypersurface de $S \times P_{1}(\mathbb{C})$, elle vérifie l'hypothèse du théorème 2.3. Alors $[\widetilde{\Gamma}]$ n'admet pas de solution au problème du bord dans $S \times P_{1}(\mathbb{C})$. En effet, si c'était le cas $\gamma$ en admettrait une aussi et donc la fonction $\mathrm{e}^{1 / z}$ admettrait une extension holomorphe au disque unité, ce qui est impossible.

Il est bien sûr nécessaire d'avoir une condition de transversalité de $\Gamma$ par rapport aux fibres du produit. En effet, si $\Gamma$ est entièrement incluse dans l'une des fibres du produit on ne peut rien conclure. Les conditions de transversalité b) et c) supposées dans le théorème sont en fait générique. D'après le théorème de Morse (voir par exemple [21]), toute fonction lisse $f: \mathbb{R}^{k} \rightarrow \mathbb{R}$ admet une déformation dont les singularités sont non dégénérées. Dans le cas d'un application à valeurs dans $\mathbb{R}^{\ell}$, ceci est encore valide. En particulier, si $\ell=k-1$ nous obtenons la proposition suivante :

BULLETIN DE LA SOCIÉtÉ MATHÉMATIQUE DE FRANCE 
Proposition 2.4. - Soit $\Gamma$ une sous-variété réelle lisse de dimension $2 n+1$ incluse dans une variété produit $U \times \omega$ où $U$ est une variété de dimension réelle $2 n$ et $\omega$ une variété réelle. Notons $\pi: U \times \omega \rightarrow U$ la projection canonique sur le premier membre. Alors il existe une déformation $\widetilde{\Gamma}$ de $\Gamma$ telle que :

1) Il existe une sous-variété $N \subset \widetilde{\Gamma}$ de dimension $2 n-1$ telle que pour tout $x \in \widetilde{\Gamma} \backslash N$, la fibre $H_{\pi(x)}=\pi^{-1}(\pi(x))$ est transverse $\grave{a} \widetilde{\Gamma}$ au point $x$.

2) Pour tout $z \in U, \sigma_{z}=N \cap H_{z}$ est une union finie de points.

3) Pour tout $z \in U, \gamma_{z}=\widetilde{\Gamma} \cap H_{z}$ est une courbe lisse par morceaux dont la partie régulière admet un nombre fini de composantes connexes.

Par conséquent, pour tout $z \in U$, la tranche $[\Gamma, \pi, z]$ est bien définie et coïncide avec $\left[\gamma_{z}\right]$, le courant d'intégration sur $\gamma_{z}$.

Exemple 2. - La sphère $S^{3}=\left\{(z, w) \in \mathbb{C} \times \mathbb{C},|z|^{2}+|w|^{2}=1\right\}$ vérifie les propriétés 1), 2) et 3) de la proposition 2.4. En effet,

1) si $|z|>1$, alors $\{z\} \times \mathbb{C}$ est transverse à $S^{3}$ et $\gamma_{z}=\varnothing$;

2) si $|z|=1$, alors $\{z\} \times \mathbb{C}$ n'est pas transverse à $S^{3}$ et $\gamma_{z}=\{(z, 0)\}$. On a $N=\left\{(z, 0) \in S^{3} ;|z|=1\right\}$;

3) si $|z|<1$, alors $\{z\} \times \mathbb{C}$ est transverse à $S^{3}$ et

$$
\gamma_{z}=\left\{(z, w) \in\{z\} \times \mathbb{C} ;|w|^{2}=1-|z|^{2}\right\} .
$$

2.2. Plan de la preuve. - Pour l'implication 1) $\Rightarrow 2$ ), nous montrerons qu'il existe un ouvert de Stein $V_{d}$ et un sous-ensemble $(n-1)$-générique $\widetilde{E}_{k, \ell}$ de $Z$ tels que pour tout $z \in \widetilde{E}_{k, \ell}$, il existe une solution $\left[S_{z}\right]$ au problème du bord pour $\left[\gamma_{z}\right]$ dont l'adhérence du support est inclus dans $V_{d}$. Ceci fait, les résultats connus sur le problème du bord dans $\mathbb{C}^{n}$ permettent de conclure que le problème du bord pour $[\Gamma]$ dans $V_{d}$ admet une solution.

L'implication 2) $\Rightarrow 3$ ) sera montrée en trois étapes. Notons $W_{\max }$ l'ensemble des $z \in U$ admettant un voisinage $W_{z}$ tel que le problème du bord pour $[\Gamma]$ ait une solution dans $W_{z} \times \omega$. Soit $z \in W_{\max }$; dans une première étape, nous montrons qu'il existe un ouvert maximal (mais non unique en général) $U_{z}$ tel que $[\Gamma]$ admette une solution $\left[T_{\max }\right]$ au problème du bord dans $U_{z} \times \omega$.

Dans la deuxième étape, nous montrons que si $[x, y] \subset U_{z}$, alors la différence de volume des courants d'intersection $\left[T_{\max }, \pi, x\right]$ et $\left[T_{\max }, \pi, y\right]$ est uniformément bornée. En particulier, en faisant tendre $y$ vers le bord de $U_{z}$, on montre qu'il existe un ensemble $(n-1)$-générique inclus dans le bord de $U_{z}$ tel que presque tous les points du bord de $U_{z}$ sont des points de $W_{\max }$.

Enfin, nous montrons par l'absurde que $U \backslash W_{\max }$ est de mesure de Hausdorff $(2 n-1)$-dimensionnelle nulle. En effet, dans le cas contraire, en partant d'un point $z_{1} \in W_{\max }$, puis d'un point $z_{2} \in \partial U_{z_{1}} \cap W_{\max }$ et ainsi de suite, on construit une suite de points $\left\{z_{i}\right\}$ convergeant vers un point du bord de $W_{\max }$. Or, à chaque étape, le volume du support des courants d'intersection $\left[T_{\max }, \pi, z_{i}\right]$ décroit d'une constante fixe ce qui est impossible pour $i$ assez grand.

TOME $130-2002-\mathrm{N}^{\mathrm{O}} 2$ 
L'implications 3) $\Rightarrow 4$ ) s'obtient en recouvrant $U \backslash F$ par une réunion localement finie de boules $\left\{B\left(z_{i}, \epsilon_{i}\right)\right\}_{i \in I}$ telles que pour tout point $z_{i} \in U \backslash F$, on ait $B\left(z_{i}, \epsilon_{i}\right) \subset O_{z_{i}}$ où $O_{z_{i}}$ est l'ouvert définit dans la proposition 3. Posons $Y=F \cup \bigcup_{i \in I} \partial B\left(z_{i}, \epsilon_{i}\right)$. Par construction, $Y$ est un fermé. Alors, pour chaque composante connexe $U_{j}(j \in J)$ de $U \backslash Y$, il existe $i \in I$ tel que $U_{j} \subset B\left(z_{i}, \epsilon_{i}\right) \subset$ $O_{z_{i}}$ et donc $[\Gamma]$ admet une solution $\left[T_{j}\right]$ au problème du bord dans $U_{j} \times \omega$. Il suffit alors de prendre pour solution au problème du bord pour $[\Gamma]$ dans $(U \backslash Y) \times \omega$ la $(n+1)$-chaîne holomorphe égale à $\left[T_{j}\right]$ dans $U_{j} \times \omega$.

L’implication 4$) \Rightarrow 1$ ) est immédiate.

2.3. Implication 1$) \Rightarrow 2$ ) : réduction au cas de $\mathbb{C}^{n}$. - L'ensemble $Z$ étant $(n-1)$-générique, il existe un point $z \in Z$ tel que l'intersection de $Z$ avec tout voisinage de $z$ est encore $(n-1)$-générique. Notons $\Pi: U \times \omega \rightarrow \omega$ la projection canonique sur le second membre. Nous rappelons que pour tout $z \in U$, nous notons $\gamma_{z}=\Gamma \cap(\{z\} \times \omega)$ et $\left[\gamma_{z}\right]$ le courant d'intégration associé. Soit $V$ un voisinage de Stein de $\Pi\left(\gamma_{z}\right)$ obtenu grâce à la proposition 5.8. Soit $V_{\epsilon} \subset \subset V$ un $\epsilon$-voisinage de $\Pi\left(\gamma_{z}\right)$ et $V_{\frac{1}{2} \epsilon} \subset \subset V_{\epsilon}$ un $\frac{1}{2} \epsilon$-voisinage de $\Pi\left(\gamma_{z}\right)$. On choisit une constante $C>0$ de sorte que tout sous-ensemble analytique irréductible de dimension 1 de $\omega \backslash \Pi\left(\bar{V}_{\frac{1}{2} \epsilon}\right)$ dont l'adhérence rencontre $\Pi\left(\bar{V}_{\frac{1}{2} \epsilon}\right)$ et $\omega \backslash \Pi\left(V_{\epsilon}\right)$, ait un volume supérieur à $C$ (voir la proposition 5.7. avec $C=C_{\epsilon / 2}^{\Pi\left(\bar{V}_{\epsilon / 2}\right)}$ ). Soit $U_{\frac{1}{2} \epsilon}$ un voisinage de Stein de $z$ dans $U$ tel que pour tout $x \in U_{\frac{1}{2} \epsilon}, \Pi\left(\gamma_{x}\right) \subset V_{\frac{1}{2}} \epsilon$. Comme $\omega$ est disque convexe, notons $\widehat{V}_{\frac{1}{2} \epsilon}$ le compact défini de manière analogue à la définition 2.1. De plus, on peut supposer que $C$ est assez petit pour que toute courbe compact incluse dans $\widehat{V}_{\frac{1}{2} \epsilon}$ ait un volume strictement supérieur à $C$. Pour tout $x \in Z$ choisissons $\left[S_{x}\right]$, une 1-chaîne holomorphe solution au problème du bord pour $\left[\gamma_{x}\right]$ minimisant le volume du support des solutions (voir proposition 5.16). Posons

$$
E_{k, \ell}=\left\{x \in Z \cap U_{\frac{1}{2} \epsilon} ; \operatorname{Vol}\left[S_{x}\right] \leq k, \frac{1}{2} \ell C<\operatorname{Vol} S_{x} \leq \frac{1}{2}(\ell+1) C\right\}
$$

où $S_{x}$ est le support de la 1-chaîne $\left[S_{x}\right]$. L'ensemble $Z \cap U_{\frac{1}{2} \epsilon}$ étant $(n-1)$-générique, il existe $k, \ell \in \mathbb{N}$ tels que $E_{k, \ell}$ soit encore $(n-1)$-générique.

LEMme 2.5. - Soit $x \in E_{k, \ell}$ et notons A l'enveloppe d'holomorphie de $\gamma_{x}$ dans $\{x\} \times V$ (qui, d'après [39], [37], [1], [28], [8] est un sous-ensemble analytique de dimension pure 1 et de volume fini de $\left.(\{x\} \times V) \backslash \gamma_{x}\right)$. Alors pour toute suite $\left\{x_{i}\right\}$ de points de $E_{k, \ell}$ convergeant vers $x$, les propriétés suivantes sont vérifiées :

1) Il existe une suite extraite de la suite $\left\{x_{i}\right\}$ (que l'on notera encore $\left\{x_{i}\right\}$ ) telle que la suite $\left\{\left[S_{x_{i}}\right]\right\}$ converge au sens des courants vers une 1-chaîne holomorphe $[S]$ solution au problème du bord pour $\left[\gamma_{x}\right]$. 
2) Quitte à extraire à nouveau une sous-suite, la suite de supports $S_{x_{i}}$ converge au sens de Bishop vers une surface de Riemann $S_{\infty}$ dont l'adhérence ne contient aucune courbe compacte.

3) $S_{\infty} \subset A \cup S$ (où $S$ est le support de $[S]$ ).

4) $S_{\infty} \subset A \cup S_{x} \cup \bigcup_{i=1}^{i=m} L_{i}$ où les $L_{i}$ sont courbes compactes contenant une composante analytique de $S_{x}$ ou de $A$.

Démonstration. - La masse des 1-chaînes holomorphes $\left[S_{x_{i}}\right]$ étant uniformément bornée, il existe une suite-extraite (que l'on notera encore $\left[S_{x_{i}}\right]$ ) qui converge vers une 1-chaîne holomorphe limite $[S]$ (voir proposition 5.6). Du fait des hypothèses de transversalité $b$ ) et $c$ ) du théorème 2.3 , la suite de bords $\left[\gamma_{x_{i}}\right]=d\left[S_{x_{i}}\right]$ converge vers le courant limite $\left[\gamma_{x}\right]=d\left[S_{x}\right]$. On a donc

$$
\left[\gamma_{x}\right]=d\left[S_{x}\right]=d[S] .
$$

Du fait que $S_{x_{i}}$ est par définition, pour tout $i \in \mathbb{N}$, relativement compact dans $\left\{x_{i}\right\} \times \omega$ et que $\omega$ est disque-convexe, on a $\Pi\left(S_{x_{i}}\right) \subset \widehat{V}_{\frac{1}{2} \epsilon}$ pour tout $i \in \mathbb{N}$ et donc $\Pi(S) \subset \widehat{V}_{\frac{1}{2} \epsilon}$. Quitte à extraire une sous-suite de $\left\{\left[S_{x_{i}}\right]\right\}$, on peut aussi supposer que la suite des supports $\left\{S_{x_{i}}\right\}$ converge au sens de Bishop vers une surface de Riemann limite $S_{\infty}$ ). On a alors :

$$
\frac{1}{2} \ell C \leq \operatorname{Vol} S_{x} \leq \operatorname{Vol} S \leq \operatorname{Vol} S_{\infty} \leq \lim _{j \rightarrow \infty} \operatorname{Vol} S_{x_{i}} \leq \frac{1}{2}(\ell+1) C .
$$

La première et deuxième inégalité vient de la définition de $E_{k, \ell}$, la troisième du fait que $S \subset S_{\infty}$, la quatrième de l'inégalité de la proposition 5.5 et enfin la dernière provient elle aussi de la définition de $E_{k, \ell}$. Par construction, $\Pi\left(S_{\infty}\right) \subset \widehat{V}_{\frac{1}{2} \epsilon}$ et toutes ses composantes irréductibles non incluses dans $V$ sont donc de volume supérieur ou égale à $C$. Par conséquent, l'inégalité précédente montre que $S_{\infty}$ ne peut contenir de composante non incluse dans $\{x\} \times V$ qui ne soit pas incluse dans $S$ et donc $S_{\infty} \subset S \cup A$. Cela montre aussi que l'adhérence de $S_{\infty}$ ne contient pas de courbe compacte. En effet, supposons le contraire. Notons $L$ une courbe compacte irréductible de $\bar{S}_{\infty}$. La courbe $L$ étant compacte, elle ne peut être incluse dans $\{x\} \times V$ et donc $L \backslash \gamma_{x}$ admet une composante irréductible $\widetilde{L} \subset S$ et dont le volume est supérieur à $C$. Mais alors $[\widetilde{S}]=[S]-n_{i}[L]$ (où $n_{i}$ est la multiplicité de $\widetilde{L}$ dans $[S]$ ) est encore une solution au problème du bord pour $\gamma_{x}$ dont le support $\widetilde{S}$ est inclus dans $S_{\infty} \backslash \widetilde{L}$ et vérifie donc :

$$
\frac{1}{2} \ell C<\operatorname{Vol} \widetilde{S} \leq \operatorname{Vol} S_{\infty}-\operatorname{Vol} \widetilde{L} \leq \operatorname{Vol} S_{\infty}-C \leq \frac{1}{2}(\ell+1) C-C
$$

ce qui donne la contradiction recherchée.

La partie régulière de la courbe $\gamma_{x}$ ayant un nombre fini de composantes, les ensembles analytiques $A$ et $S$ admettent un nombre fini de composantes irréductibles. Pour la dernière propriété, il suffit de prendre pour $\left\{L_{i}\right\}$ l'ensemble

TOME $130-2002-\mathrm{N}^{\mathrm{O}} 2$ 
des courbes irréductibles compactes contenant l'une de ces composantes. En effet, comme $S_{\infty} \subset A \cup S$, il suffit de montrer que

$$
A \cup S \subset A \cup S_{x} \cup \bigcup_{i=1}^{i=m} L_{i} .
$$

Les 1-chaînes holomorphes $[S]$ et $\left[S_{x}\right]$ sont toutes deux solution au problème du bord pour $\left[\gamma_{x}\right]$. Par conséquent, le courant $[R]=[S]-\left[S_{x}\right]$ est une 1-chaîne holomorphe fermée de $\{x\} \times \omega$ dont le support est compact. On a donc

$$
[R]=\sum_{j \in J} n_{j}\left[R_{j}\right]
$$

où les $\left[R_{j}\right]$ sont des courants d'intégration sur des courbes compactes irréductibles $R_{j}$ de $\{x\} \times \omega$. Par conséquent, pour conclure il reste à montrer que pour tout $j \in J$, il existe $i \in\{1, \ldots, m\}$ tel que $R_{j} \subset L_{i}$. Or, par construction $S \subset S_{\infty}$ et $\bar{S}$ ne contient aucune courbe compacte. Par conséquent, pour tout $j \in J$, la courbe $R_{i}$, contient au moins une composante irréductible de $S_{x}$ et est donc incluse dans l'une des courbes $L_{i}$.

On choisit un point $x$ de $E_{k, \ell}$ tel que $E_{k, \ell}$ soit $(n-1)$-générique dans tout voisinage de $x$ et notons $A$ l'enveloppe d'holomorphie de $\gamma_{x}$ dans $\{x\} \times V$. Soit $L_{1}, \ldots, L_{m}$, l'ensemble des courbes compactes irréductibles contenant une composante analytique de $S_{x} \cup A$. Pour tout $i \in\{1, \ldots, m\}$ notons $L_{i}^{j}$, $j \in\left\{1, \ldots, p_{i}\right\}$ les composantes irréductibles de $L_{i} \backslash \gamma_{x}$. Posons

$$
D=\left\{1, \ldots, p_{1}\right\} \times \cdots \times\left\{1, \ldots, p_{m}\right\}
$$

et pour tout $d=\left(d_{1}, \ldots, d_{m}\right) \in D$, définissons

$$
S_{d}=\left(S_{x} \cup A \cup \bigcup_{i=1}^{i=m} L_{i}\right) \backslash\left(\bigcup_{i=1}^{i=m} L_{i}^{d_{i}}\right) .
$$

Par construction, $S_{d}$ est une surface de Riemann ne contenant pas de courbes compactes et pour toute suite de points $x_{i} \in E_{k, \ell}$, il existe un $d \in D$ tel que la surface $S_{\infty}$ soit incluse dans $S_{d}$ où $S_{\infty}$ est construite dans le lemme 2.5.

Pour tout $d \in D$, fixons $V_{d}$ un voisinage de Stein de $S_{d} \cup \gamma_{x}$ (voir prop. 5.8).

Lemme 2.6. - Il existe $d \in D$ et un sous-ensemble $(n-1)$-générique $\widetilde{E}_{k, \ell} \subset E_{k, \ell}$ tels que $S_{w} \cup \gamma_{x} \subset V_{d}$ pour tout $w \in \widetilde{E}_{k, \ell}$.

Démonstration. - Pour tout $d \in D$, définissons

$$
E_{k, \ell}^{d}=\left\{w \in E_{k, \ell} ; S_{w} \subset V_{d}\right\}
$$

et montrons qu'au moins l'un de ces ensembles est $(n-1)$-générique. Procédons par l'absurde et supposons que pour tout $d \in D$, l'ensemble $E_{k, \ell}^{d}$ est non $(n-1)$ générique. Mais alors l'ensemble $\bigcup_{d \in D} E_{k, \ell}^{d}$ serait lui aussi non $(n-1)$-générique 
en tant que réunion finie de tels ensembles. L'ensemble $E=E_{k, \ell} \backslash \bigcup_{d \in D} E_{k, \ell}^{d}$ est alors $(n-1)$-générique dans tout voisinage de $x$. Appliquons le lemme 2.5 à une suite de points $x_{i} \in E$ convergeant vers $x$. Il existe donc $d \in D$ telle que la suite converge vers une limite $S_{\infty} \subset S_{d}$. Mais alors par définition de la notion de convergence au sens de Bishop, pour $i$ assez grand $S_{x_{i}} \subset V_{d}$ et donc pour $i$ assez grand $x_{i} \in E_{k, \ell}^{d}$. Ce qui donne la contradiction recherchée.

Nous avons donc construit un voisinage de Stein $V_{d}$ de $\gamma_{x}$ et un ensemble $(n-1)$-générique $\widetilde{E}_{k, \ell} \subset U$ tels que pour tout $w \in \widetilde{E}_{k, \ell}$, la tranche $\left[\gamma_{w}\right]=[\Gamma, \Pi, w]$ admette une solution au problème du bord dont le support est inclus dans $V_{d}$. Soit $O \subset U_{\frac{1}{2} \epsilon}$ un voisinage de Stein de $x$. Alors $O \times V_{d}$ est le produit de deux variétés de Stein et la résolution du problème du bord dans $\mathbb{C}^{n}$ (voir proposition 5.2) montre que $[\Gamma]$ admet une solution au problème du bord dans $O \times V_{d}$ et donc dans $O \times \omega$ ce qui termine la preuve de l'implication.

\subsection{Implication 2) $\Rightarrow 3$ ) : contrôle du volume jusqu'au bord}

Supposons que le problème du bord pour $[\Gamma]$ admet une solution $[T]$ dans $W \times \omega$ où $W$ est un ouvert relativement compact assez petit de $U$. Soit $\widetilde{W} \subset U$ un voisinage de Stein de $\bar{W}$. Alors $\widetilde{W} \times \omega$ est encore une variété kählérienne. On montre qu'il existe une majoration du volume des 1-chaînes holomorphes $\left[S_{z}\right]=[T, \pi, z]$ quand $z$ approche du bord de $W$ ne dépendant que de la géométrie de $\Gamma$ :

Lemme 2.7. - Soit $\left(x_{1}, \ldots, x_{2 n}\right)$ un système de coordonnées réelles de $\widetilde{W}$. Soit $\Psi: W \rightarrow \mathbb{R}^{2 n-1}$ la projection $\Psi\left(x_{1}, \ldots, x_{2 n}\right)=\left(x_{2}, \ldots, x_{2 n}\right)$. D'après le théorème de Sard appliqué à l'application $\Psi \circ \pi$, il existe un ensemble $K \subset \mathbb{R}^{2 n-1}$ de mesure nulle tel que pour tout $X \in \mathbb{R}^{2 n-1} \backslash K, \Gamma$ est transverse à la variété $\pi^{-1}\left(\Psi^{-1}(X)\right)$. Pour tout $X \in \mathbb{R}^{2 n-1} \backslash K$ et pour tous $a, b \in \mathbb{R}$, on a alors les inégalités suivantes :

$$
\begin{aligned}
\left|\operatorname{Vol}\left(\left[S_{(a, X)}\right]\right)-\operatorname{Vol}\left(\left[S_{(b, X)}\right]\right)\right| & \leq M_{W} \cdot \mathcal{H}_{2}\left(\Gamma \cap \pi^{-1}([(a, X),(b, X)])\right) \\
& \leq M_{W} \cdot \mathcal{H}_{2}\left(\Gamma \cap \pi^{-1}\left(\Psi^{-1}(X)\right)\right), \\
\left|\operatorname{Vol}\left(S_{(a, X)}\right)-\operatorname{Vol}\left(S_{(b, X)}\right)\right| & \leq M_{W} \cdot \mathcal{H}_{2}\left(\Gamma \cap \pi^{-1}([(a, X),(b, X)])\right)
\end{aligned}
$$

où $M_{W}=\sup _{(W \times \omega) \cap \Gamma}|\Omega|$ pour la forme kählérienne $\Omega$ associée à la variété $\widetilde{W} \times \omega$ et où $\mathcal{H}_{p}$ est le volume (ou mesure de Hausdorff) p-dimensionnelle sur $U \times \omega$.

Démonstration. - Comme l'ouvert $W$ est relativement compact dans $\widetilde{W}$, d'après la propriété a) du théorème $2.3, \pi^{-1}(\bar{W}) \cap \Gamma$ est un compact de $\widetilde{W} \times \omega$ et donc $M_{W}$ est fini. Par définition, on a

$$
[T]=\sum n_{i}\left[T_{i}\right]
$$

TOME $130-2002-\mathrm{N}^{\mathrm{O}} 2$ 
où $n_{i} \in \mathbb{Z}$ et $\left[T_{i}\right]$ sont les courants d'intégration sur des sous-ensemble analytiques de dimension $(n+1)$ de $W \times \omega \backslash \Gamma$. On a alors par hypothèse

$$
[\Gamma]=\sum n_{i} d\left[T_{i}\right]
$$

Soient

$$
\left[T^{+}\right]=\sum\left|n_{i}\right|\left[T_{i}\right], \quad\left[\Gamma^{+}\right]=\sum\left|n_{i}\right| d\left[T_{i}\right],
$$

$\Gamma_{i}$ le bord orienté de $T_{i}$ (c'est-à-dire $\left[\Gamma_{i}\right]=d\left[T_{i}\right]$ ) et $M_{i}$ les composantes connexes de $\Gamma \cap W \times \omega$. On a alors d'après le théorème de Stokes

$$
\begin{aligned}
0 & =\sum\left|n_{i}\right| \int_{T_{i} \cap \pi^{-1}([(a, X),(b, X)])} \mathrm{d} \Omega \\
& =\sum\left|n_{i}\right|\left(\int_{\left(\Gamma_{i} \cap \pi^{-1}([(a, X),(b, X)])\right.} \Omega+\int_{T_{i} \cap \pi^{-1}((b, X))} \Omega-\int_{T_{i} \cap \pi^{-1}((a, X))} \Omega\right) \\
& =\left(\sum\left|n_{i}\right| \int_{\left(\Gamma_{i} \cap \pi^{-1}([(a, X),(b, X)])\right.} \Omega\right)+\operatorname{Vol}\left(\left[S_{(b, X)}\right]\right)-\operatorname{Vol}\left(\left[S_{(a, X)}\right]\right) .
\end{aligned}
$$

En regroupant les composantes $M_{j}$ des $\Gamma_{i}$, et en tenant compte de l'orientation, on a

$$
\begin{aligned}
\left.\left|\sum\right| n_{i} \mid \int_{\left(\Gamma_{i} \cap \pi^{-1}([(a, X),(b, X)])\right.} \Omega\right) \mid & =\left|\sum \pm \int_{M_{j} \cap \pi^{-1}([(a, X),(b, X)])} \Omega\right| \\
& \leq\left|\int_{\Gamma \cap \pi^{-1}([(a, X),(b, X)])}\right| \Omega|| \\
& \leq M_{W} \mathcal{H}_{2}\left(\Gamma \cap \pi^{-1}([(a, X),(b, X)])\right) \\
& \leq M_{W} \mathcal{H}_{2}\left(\Gamma \cap \pi^{-1}\left(\Psi^{-1}(X)\right)\right),
\end{aligned}
$$

ce qui termine la démonstration de la première inégalité.

La deuxième inégalité s'obtient alors en appliquant à la chaîne $[\widetilde{T}]=\sum\left[T_{i}\right]$ ce qui précède.

On note $W_{\max }$ l'ensemble des points $z \in U$ possédant un voisinage $W_{z} \subset U$ tel que le problème du bord pour [ $[\Gamma]$ soit résoluble dans $W_{z} \times \omega$. Bien sûr $W_{\max }$ est un ouvert de $U$.

Lemme 2.8. - Soient $z \in W_{\max }$ et $W_{z}$ un voisinage connexe de $z$ dans $U$ tel que le problème du bord pour $[\Gamma]$ admette une solution $[T]$ dans $W_{z} \times \omega$. Alors il existe un ouvert connexe maximal $U_{z} \subset W_{\max }$ tel que le problème du bord pour $[\Gamma]$ ait une solution $\left[T_{\max }\right]$ dans $U_{z} \times \omega$ coïncidant avec $[T]$ dans $W_{z} \times \omega$. De plus, $\partial U_{z} \backslash W_{\max }$ est un fermé de mesure de Hausdorff $(2 n-1)$-dimensionnelle nulle. 
Démonstration. - D'après le lemme de Zorn, pour montrer l'existence de $U_{z}$, il suffit de montrer que si $U_{1}$ et $U_{2}$ sont deux ouverts connexes de $U$ contenant $W_{z}$, tels que $U_{1} \subset U_{2}$ et tels que la solution $\left[T_{U_{1}}\right]$ (resp. $\left[T_{U_{2}}\right]$ ) au problème du bord pour $[\Gamma]$ dans $U_{1} \times \omega\left(\right.$ resp. $\left.U_{2} \times \omega\right)$ coïncide avec $[T]$ dans $W_{z} \times \omega$, alors $\left[T_{U_{2}}\right]$ coïncide avec $\left[T_{U_{1}}\right]$ dans $U_{1} \times \Omega$. La $n$-chaîne holomorphe $[L]=\left[T_{U_{2}}\right]-\left[T_{U_{1}}\right]$ est bien définie dans $\left(U_{1} \times \omega\right) \backslash \Gamma$. Par hypothèse, $[L]$ est fermée et $[L]=0$ dans $W_{z} \times \omega$. Le support $L$ de $[L]$ est donc un sous-ensemble analytique de $U_{1} \times \omega$ dont l'intersection avec $W_{z} \times \omega$ est vide. Soit $\pi: U \times \omega \rightarrow U$ la projection canonique sur $U$. Par construction, si $L$ n'est pas vide, il contient une composante connexe $\Gamma_{i}$ de $\Gamma \cap U_{1} \times \omega$ et donc $\pi(L) \supset \pi\left[\Gamma_{i}\right]$ qui contient un ouvert de $U_{1}$ d'après les hypothèses de transversalité faites sur $\Gamma$. D'après le théorème de l'application propre, la projection $\pi(L)$ de $L$ sur $U_{1}$ est donc soit vide (si $L$ l'est) soit tout $U_{1}$ car $U_{1}$ est connexe. Or, comme $L \cap W_{z} \times \omega=\varnothing$, $L$ est nécessairement vide dans $U_{1} \times \omega$ et donc $\left[T_{U_{2}}\right]=\left[T_{U_{1}}\right]$ dans $U_{1} \times \omega$, d'où l'existence de $U_{z}$.

Supposons que l'ensemble $G_{z}=\partial U_{z} \backslash W_{\max }$ est un fermé de mesure de Hausdorff $(2 n-1)$-dimensionnelle non nulle. On choisit un point $x_{0} \in G_{z}$ tel que $G_{z}$ soit de mesure non nulle dans tout voisinage $x_{0}$. Choisissons alors un voisinage $W_{x_{0}}$ de $x_{0}$ et un système de coordonnées $\left(x_{1}, \ldots, x_{2 n}\right)$ de $W_{x_{0}}$ tels que :

1) l'ensemble $H=\Psi\left(G_{z} \cap W_{x_{0}}\right)$ soit de mesure non nulle dans $\mathbb{R}^{2 n-1}$ où $\Psi: \mathbb{R}_{+} \times \mathbb{R}^{2 n-1} \rightarrow \mathbb{R}^{2 n-1}$ est la projection $\Psi\left(x_{1}, \ldots, x_{2 n}\right)=\left(x_{2}, \ldots, x_{2 n}\right) ;$

2) l'ensemble $\{0\} \times \mathbb{R}^{2 n-1}$ est contenu dans $W_{x_{0}}$.

D'après le théorème de Sard, pour presque tout $X \in \mathbb{R}^{2 n-1}$, le courant d'intersection $[\Gamma, \Pi, X]$ est bien défini. Quitte à restreindre $H$, on peut supposer que ceci est vérifié pour tous les points de $H$. Soit

$$
\widetilde{H}=\left\{\left(\lambda_{X}, X\right) \in \overline{\mathbb{R}}_{+} \times H ; \lambda_{X}=\sup \left\{t \in \mathbb{R}_{+} ;\left[0, t\left[\times\{X\} \subset W_{x_{0}}\right\}\right\} .\right.\right.
$$

Soit $\left(\lambda_{X}, X\right) \in \widetilde{H}$. Par définition de $H, \lambda_{X}<\infty$; d'après le lemme 2.7, le volume des 1-chaînes holomorphes $\left[S_{(t, X)}\right]=[T, \pi,(t, X)]$ (où $\pi: U \times \omega \rightarrow U$, $\pi(x, w)=x)$ pour $t<\lambda_{X}$ est uniformément borné. D'après la proposition 5.6, il existe alors une suite de réels $\left\{t_{i}\right\}$ convergeant vers $\lambda_{X}$ telle que la suite de 1-chaînes holomorphes $\left[S_{\left(t_{i}, X\right)}\right]$ converge au sens des courants vers une 1chaîne holomorphe $\left[S_{(\lambda, X)}\right]$ solution au problème du bord pour $\left[\gamma_{(\lambda, X)}\right]$. On a donc $\widetilde{H} \subset G_{z}$ et est $(n-1)$-générique (car de mesure de Hausdorff $(2 n-1)$ dimensionnelle non nulle). L'implication 1) $\Rightarrow 2$ ) avec $Z=\widetilde{H}$ permet alors d'obtenir la contradiction recherchée.

Soit $G=\partial W_{\max }$. Il reste à montrer que $G$ est de mesure de Hausdorff $(2 n-1)$-dimensionnelle nulle. En effet, si c'est le cas on aura $G=U \backslash W_{\max }$ car $U$ est connexe et un ensemble de mesure de Hausdorff $(2 n-1)$-dimensionnelle nulle ne peut déconnecter un ouvert de dimension $2 n$. Procédons par l'absurde et supposons que $G$ est de mesure $(2 n-1)$-dimensionnelle non nulle. Il existe alors

TOME $130-2002-\mathrm{N}^{\mathrm{O}} 2$ 
un point $z \in G$ tel que pour tout voisinage $W_{z}$ de $z$ dans $U$, l'ensemble $W_{z} \cap G$ soit de mesure de Hausdorff $(2 n-1)$-dimensionnelle non nulle.

Soit $\Pi: U \times \omega \rightarrow \omega$ la projection sur le second membre. Soit $V$ un voisinage de Stein de $\Pi\left(\gamma_{z}\right)=\Pi(\Gamma \cap\{z\} \times \omega)$. Posons

$$
C=C_{\frac{1}{2} \epsilon}^{\overline{\bar{V}_{\frac{1}{2}} \epsilon}}>0
$$

la constante définie dans la proposition 5.7 avec $\epsilon$ choisi de manière à ce que le voisinage $V_{\epsilon}$ soit inclus dans $V$. Par conséquent, tout sous-ensemble analytique irréductible de dimension 1 de $V \backslash \bar{V}_{\frac{1}{2} \epsilon}$ dont l'adhérence rencontre $\bar{V}_{\frac{1}{2} \epsilon} V \backslash \bar{V}_{\epsilon}$, a un volume strictement supérieur à $C$. Soit $x_{0} \in W_{\max }$ tel que $\gamma_{x_{0}} \subset\left\{x_{0}\right\} \times V_{\epsilon}$. Soient $\widetilde{W}_{z}$ et $W_{z} \subset \subset \widetilde{W}_{z}$ deux voisinages de Stein de $z$ dans $U$ et $\left(x_{1}, \ldots, x_{2 n}\right)$ un système de coordonnées de $W_{z}$ tels que :

1) pour tout $x \in W_{z}, \Pi\left(\gamma_{x}\right) \subset V_{\frac{1}{2} \epsilon}$;

2) l'ensemble $H=\Psi\left(G \cap V_{z}\right)$ soit de mesure non nulle dans $\mathbb{R}^{2 n-1}$ où $\Psi$ : $\mathbb{R}_{+} \times \mathbb{R}^{2 n-1} \rightarrow \mathbb{R}^{2 n-1}$ est la projection $\Psi\left(x_{1}, \ldots, x_{2 n}\right)=\left(x_{2}, \ldots, x_{2 n}\right) ;$

3) l'ensemble $\{0\} \times \mathbb{R}^{2 n-1} \subset U_{x_{0}} \cap W_{z}$ où $U_{x_{0}}$ est un ouvert maximal contenant $x_{0}$ tel que le problème du bord pour $[\Gamma]$ admet une solution $\left[T_{x_{0}}\right]$ dans $U_{x_{0}} \times \omega$;

4) $W_{z}$ est assez petit pour que si $X \in \mathbb{R}^{2 n-1}$ on ait

$$
M_{W_{z}} \cdot \operatorname{Vol}\left(\Gamma \cap \pi^{-1}\left(\Psi^{-1}(X)\right)\right)<\frac{1}{2} C
$$

où $M_{W_{z}}=\sup _{\Gamma \cap \pi^{-1}\left(W_{z}\right)}|\Omega|$ avec $\Omega$ la forme kählérienne sur $\widetilde{W} \times \omega$.

D'après le théorème de Sard, il existe un fermé $E \subset \mathbb{R}^{2 n-1}$ de mesure nulle tel que pour tout $X \in \mathbb{R}^{2 n-1} \backslash E$, la variété $\pi^{-1}\left(\Psi^{-1}(X)\right)$ soit transverse à $\Gamma$. Le courant d'intersection est donc bien défini (on le notera $[\Gamma, \Psi \circ \pi, X]$ ). Quitte à remplacer l'ouvert $W_{z}$ par un ouvert de $W_{z} \backslash E$, on peut toujours supposer que $W_{z} \subset \mathbb{R}^{n} \backslash E$. L'ensemble $H$ étant de mesure de Hausdorff $(2 n-1)$ dimensionnelle non nulle (et est donc $(n-1)$-générique), il existe $k, \ell \in \mathbb{N}$ tels que l'ensemble

$$
H_{\ell}^{0}=\left\{(0, X) \in W_{z} ; \operatorname{Vol} S_{(0, X)}<k \text { et } \operatorname{Vol}\left[S_{(0, X)}\right]<\ell\right\}
$$

(où $\left.\left[S_{(0, X)}\right]=\left[T_{x_{0}}, \pi,(0, X)\right]\right)$ soit encore de mesure de Hausdorff $(2 n-1)$ dimensionnelle non nulle. Pour tout $X \in \Psi\left(H_{\ell}^{0}\right)$, notons $\lambda_{X}$ la borne supérieure des réels $t$ pour lesquels il existe un voisinage $V_{(t, X)}$ de $[0, t[\times\{X\}$ tels que le problème du bord pour $[\Gamma]$ admette une solution $\left[T_{(t, X)}\right]$ dans $V_{(t, X)} \times \omega$ qui coïncide avec $\left[T_{x_{0}}\right]$ au voisinage de $(0, X) \times \omega$. Soient $X \in \Psi\left(H_{\ell}^{0}\right)$, $0<t_{1}<t_{2}<\cdots<t_{n}<\cdots<\lambda_{X}$ une suite de réels convergeant vers $\lambda_{X}$ et

$$
\left[S_{n}\right]=\left[T_{\left(t_{n}, X\right)}, \pi,\left(t_{n}, X\right)\right] .
$$


D'après le lemme 2.7, pour tout $X \in \Psi\left(H_{\ell}^{0}\right)$,

$$
\begin{aligned}
\operatorname{Vol}\left[S_{n}\right] & \leq \operatorname{Vol}\left[S_{(0, X)}\right]+M_{W_{z}} \cdot \operatorname{Vol}\left(\Gamma \cap\left[(0, X),\left(t_{n}, X\right)\right] \times \omega\right) \\
& <\ell+M_{W_{z}} \cdot \operatorname{Vol}\left(\Gamma \cap\left[(0, X),\left(\lambda_{X}, X\right)\right] \times \omega\right), \\
\operatorname{Vol} S_{n} & \leq \operatorname{Vol} S_{(0, X)}+M_{W_{z}} \cdot \operatorname{Vol}\left(\Gamma \cap\left[(0, X),\left(t_{n}, X\right)\right] \times \omega\right) \\
& <k+M_{W_{z}} \cdot \operatorname{Vol}\left(\Gamma \cap\left(\left[(0, X),\left(\lambda_{X}, X\right)\right] \times \omega\right)\right) .
\end{aligned}
$$

On en déduit qu'il existe une suite extraite (que l'on notera encore $\left\{\left[S_{n}\right]\right\}$ ) convergeant au sens des courants vers une 1-chaîne limite $\left[S^{\infty}\right]$ solution au problème du bord pour $\left[\gamma_{\left(\lambda_{X}, X\right)}\right]$ et telle que la suite des supports $S_{n}$ converge au sens de Bishop vers une surface de Riemann limite $S_{\infty}$. L'ensemble $\gamma_{\left(\lambda_{X}, X\right)} \cup S_{\infty}$ contient alors nécessairement une surface de Riemann compacte $L$. En effet, sinon, en considérant un voisinage de Stein $V_{X}$ de $\gamma_{\left(\lambda_{X}, X\right)} \cup S_{\infty}$, on montre grâce à la proposition 5.2 qu'il existe un voisinage $\Lambda$ de $\left(\lambda_{X}, X\right)$ tel que $[\Gamma]$ admette une solution au problème du bord dans $\Lambda \times \omega \cap V_{X}$ qui coïncide avec $\left[T_{\left(t_{n}, X\right)}\right]$ (pour un $n$ assez grand) là où les deux solutions sont définies simultanément. Par conséquent cela montre que $[\Gamma]$ admet une solution au problème du bord au voisinage de $\left[(0, X),\left(\lambda_{X}, X\right)\right] \times \omega$ ce qui contredit la définition de $\lambda_{X}$. Mais alors, il existe $m \in \mathbb{Z}$ tel que la 1-chaîne holomorphe $\left[S^{\infty}\right]-m[L]$ soit une solution au problème du bord pour $\left[\gamma_{\left(\lambda_{X}, X\right)}\right]$ à support dans $S_{\infty} \backslash \widetilde{L}$ où $\widetilde{L}$ est une composante irréductible de $L \backslash \gamma_{\left(\lambda_{X}, X\right)}$ dont le volume est strictement supérieur à $C$. Soit $\left[A_{\left(\lambda_{X}, X\right)}\right]$ une solution au problème du bord pour $\left[\gamma_{\left(\lambda_{X}, X\right)}\right]$ ayant un support de volume minimal (voir proposition 5.16). On a donc, pour tout $X \in \Psi\left(H_{\ell}^{0}\right)$,

$$
\operatorname{Vol} A_{\left(\lambda_{X}, X\right)}<k+M_{W_{z}} \cdot \operatorname{Vol}\left(\Gamma \cap\left[(0, X),\left(\lambda_{X}, X\right)\right] \times \omega\right)-C<k-\frac{1}{2} C .
$$

L'ensemble $H_{\ell}^{0}$ étant $(n-1)$-générique, d'après l'implication 1$\left.) \Rightarrow 2\right)$, il existe un point $x_{1}=\left(\lambda_{X}, X\right)$ où $(0, X) \in H_{\ell}^{0}$ tel que le problème du bord pour $[\Gamma]$ admette une solution au dessus d'un voisinage $W_{x_{1}}$ de $x_{1}$ et tel que l'ensemble des points de la forme $\left(\lambda_{Y}, Y\right)$ avec $Y \in \Psi\left(H_{\ell}^{0}\right)$ est encore $(n-1)$-générique dans tout voisinage de $x_{1}$. On a bien sûr $W_{x_{1}} \subset W_{\max }$. En appliquant le même raisonnement que précédemment à $W_{x_{1}}$, on construit un ensemble de mesure de Hausdorff $(2 n-1)$-dimensionnelle non nulle $H_{\ell_{1}}^{1}$ tel que pour tout $\left(\lambda_{X}, X\right) \in H_{\ell_{1}}^{1}$ on ait

$$
\operatorname{Vol} A_{\left(\lambda_{X}, X\right)} \leq k-\frac{1}{2} C+M_{W_{z}} \cdot \operatorname{Vol}\left(\Gamma \cap \pi^{-1}\left(\Psi^{-1}(X)\right)\right)-C<k-2 \frac{1}{2} C
$$

et par récurrence, on construit une suite d'ensembles $H_{\ell_{n}}^{n}$ tels que pour tout $\left(\lambda_{X}, X\right) \in H_{\ell_{n}}^{n}$, on ait

$$
\operatorname{Vol} A_{\left(\lambda_{X}, X\right)} \leq k-\frac{1}{2}(n+1) C .
$$

TOME $130-2002-\mathrm{N}^{\mathrm{O}} 2$ 
Pour $n$ assez grand, $k-\frac{1}{2}(n+1) C<0$ et nous obtenons la contradiction recherchée. Ce qui termine la preuve du théorème 2.3 .

\section{Problème du bord dans les variétés produit : cas rectifiable}

La régularité de $\Gamma$ dans le théorème principal ne joue pas un rôle important. En effet, le théorème est encore valide dans le cadre des courants rectifiables (voir [14], [8]) dont le support est est géométriquement m-rectifiable ou encore est de classe $A_{m}$. Rappelons que $A$ est géométriquement $m$-rectifiable si $A$ est $\left(\mathcal{H}_{m}, m\right)$-rectifiable et si le cône tangentiel de $A$ en $\mathcal{H}_{m}$-presque tout point est un espace vectoriel réel de dimension $m$ avec $\mathcal{H}_{m}$ la mesure de Hausdorff de dimension $m$.

Soient $X, Y$ deux variétés réelles lisses, $Y$ de dimension $p \leq m$ et $f: X \rightarrow Y$ une application $C^{\infty}$ et $\Gamma$ un courant rectifiable de dimension $m$. Alors pour $\mathcal{H}_{p}$-presque tout $y \in Y$, la tranche $[\Gamma, f, y]$ est un courant rectifiable de dimension $m-p$ de support inclus dans $\Gamma \cap f^{-1}(y)$ vérifiant :

$$
\int_{Y} \Phi(y)([\Gamma, f, y], \Psi) \cdot \Omega=\left[\Gamma, f^{*}(\Phi \cdot \Omega) \wedge \Psi\right]
$$

où $\Psi$ est une $(m-p)$-forme à support compact, $\Phi$ une fonction à support compact et $\Omega$ est la forme volume de $Y$ (voir [14]).

Un $(2 p-1)$-courant $[\Gamma]$ de $\mathbb{C}^{n}$ est dit maximalement complexe s'il admet une décomposition

$$
[\Gamma]=[\Gamma]_{p, p-1}+[\Gamma]_{p-1, p}
$$

où $[\Gamma]_{p, p-1}$ et $[\Gamma]_{p-1, p}$ sont respectivement des courants de bidimension $(p, p-1)$ et $(p-1, p)$. Bien sûr, une variété lisse $\Gamma$ est maximalement complexe si et seulement si le courant d'intégration $[\Gamma]$ associé est maximalement complexe.

Dans toute la suite, nous supposerons que $[\Gamma]$ est un courant rectifiable, fermé et maximalement complexe de $U \times \omega$ dont le support $\Gamma$ est de classe $A_{2 n+1}$. Pour tout $z \in U$, nous noterons $\left[\gamma_{z}\right]$ la tranche $[\Gamma, \pi, z]$ quand elle est bien définie. Nous noterons aussi $\gamma_{z}=\Gamma \cap\{z\} \times \omega$. Le support de $\left[\gamma_{z}\right]$ est bien sûr inclus dans $\gamma_{z}$ mais la réciproque n'est pas toujours vraie.

THÉORÈmE 3.1. - Soient $U$ une variété complexe connexe de dimension $n$, $\omega$ une variété kählérienne disque convexe et $\pi: U \times \omega \rightarrow U$ la projection $(z, w) \mapsto z$. Soit $[\Gamma]$ un courant rectifiable, fermé et maximalement complexe de $U \times \omega$ dont le support $\Gamma$ est de classe $A_{2 n+1}$ et vérifie que pour tout compact $K \subset U, \Gamma \cap \pi^{-1}(K)$ est un compact de $U \times \omega$. Supposons que pour toute 2 -forme lisse $\Theta$ définie sur $\omega$, on ait $[\Gamma] \wedge \Theta=0$. Alors les propositions suivantes sont équivalentes :

1) Il existe un sous-ensemble $(n-1)$-générique $Z$ de $U$ tel que pour tout $z \in Z$, la tranche $\left[\gamma_{z}\right]=[\Gamma, \pi, z]$ est bien définie et il existe une 1-chaîne 
holomorphe $\left[S_{z}\right]$ de masse finie de $(\{z\} \times \omega) \backslash \gamma_{z}$, dont l'adhérence du support $S_{z}$ est un compact de $\{z\} \times \omega$ et telle que $d\left[S_{z}\right]=\left[\gamma_{z}\right]$.

2) Il existe un ouvert non vide $O \subset U$ tel que $[\Gamma]$ admette une solution au problème du bord dans $O \times \omega$ (i.e. il existe une $(n+1)$-chaîne holomorphe $[T]$ de $(O \times \omega) \backslash \Gamma$, de masse localement finie dans $O \times \omega$ et telle que pour tout compact $K \subset O, T \cap \pi^{-1}(K)$ est relativement compact dans $O \times \omega$ et $d[T]=[\Gamma]$ dans $O \times \omega)$.

De plus si l'on suppose que pour tout $z \in U$ la tranche $\left[\gamma_{z}\right]=[\Gamma, \pi, z]$ est bien définie et est un 1-courant rectifiable fermé dont le support est de classe $A_{1}$. Alors les deux propositions précédentes sont aussi équivalentes aux propositions suivantes :

3) Il existe un fermé $G \subset U$ de mesure de Hausdorff $(2 n-1)$-dimensionnelle nulle tel que $\forall z \notin G$, il existe un voisinage $W_{z}$ de $z$ dans $U$ tel que le problème du bord pour $[\Gamma]$ soit résoluble dans $W_{z} \times \omega$.

4) Il existe un fermé $F$ de mesure nulle dans $U$ et une $(n+1)$-chaîne holomorphe $T$ de $((U \backslash F) \times \omega) \backslash \Gamma$ telle que le problème du bord pour $[\Gamma]$ soit résoluble dans $(U \backslash F) \times \omega$.

La preuve étant en presque tous points identique à celle du cas lisse, nous signalons juste les points qui diffèrent (une démonstration complète se trouve dans [34]).

Dans la démonstration du cas lisse, un point important est l'utilisation du théorème de Sard. Dans le cas d'ensembles géométriquement rectifiables, ce dernier peut être remplacé par la proposition suivante :

Proposition 3.2 (voir [8], lemme 1.4). - Soit [Г] un courant rectifiable de $\mathbb{C}^{m+n}$ dont le support $\Gamma$ est de classe $A_{2 n+1}$. Alors, pour presque tous les $m$ plans $\mathbb{C}_{\nu}^{n} \subset \mathbb{C}^{n+m}$ et pour presque tous les points $z \in \mathbb{C}_{\nu}^{n}$, la tranche $\left[\Gamma, \pi_{\nu}, z\right]$ (où $\pi_{\nu}$ est la projection orthogonale sur $\mathbb{C}_{\nu}^{n}$ ) est un courant rectifiable dont le support est de classe $A_{1}$.

Soient $\gamma \subset \mathbb{C}^{n}$ une compact de classe $A_{1}$ et $\widehat{\gamma}$ son enveloppe d'holomorphie. D'après [39], [37], [1], [28], [8], l'ensemble $A=\widehat{\gamma} \backslash \gamma$ est un sous-ensemble analytique de dimension pure 1 et de volume fini de $\mathbb{C}^{n} \backslash \gamma$. Dans le cas où $\gamma$ est une courbe lisse par morceaux dont la partie régulière admet un nombre fini de composantes connexes, $A$ admet un nombre fini de composantes irréductibles. Cette dernière propriété a été utilisée à de nombreuses reprises dans la preuve du théorème 2.3 afin de montrer qu'il existe qu'un ensemble fini de courbes compactes irréductibles $L_{1}, \ldots, L_{k}$ contenant l'une des composantes irréductibles de $A$ (voir par exemple le lemme 2.5).

Comme le montre l'exemple suivant, ceci n'est plus vrai dans le cas où $\gamma$ est un compact de classe $A_{1}$ :

TOME $130-2002-\mathrm{N}^{\mathrm{O}} 2$ 
Exemple 3. - Pour tout $i \in \mathbb{N}^{*}$, définissons $z_{i}=1 / i$,

$$
\begin{aligned}
D_{i} & =\left\{\left(z_{i}, z\right) \in \mathbb{C}^{2} ;|z|<\frac{1}{2^{n}}\right\}, \\
C_{i}=\partial D_{i}^{+} & =\left\{\left(z_{i}, z\right) \in \mathbb{C}^{2} ;|z|=\frac{1}{2^{n}}\right\} .
\end{aligned}
$$

Notons $\left[D_{i}^{+}\right]$et $\left[C_{i}\right]$ les courants d'intégration sur $D_{i}^{+}$et $C_{i}$ orientés de manière à ce que $\left[C_{i}\right]=d\left[D_{i}^{+}\right]$et $\gamma=\bigcup_{i=1}^{\infty} C_{i} \cup\{(0,0)\}$. Alors,

$$
[S]=\sum_{i=1}^{\infty}\left[D_{i}^{+}\right]
$$

est une 1-chaîne holomorphe de masse finie de $\mathbb{C}^{2} \backslash \gamma$. Son bord $[\gamma]=\sum_{i=1}^{\infty}\left[C_{i}\right]$ est un 1-courant rectifiable dont le support $\gamma$ est de classe $A_{1}$.

Dans ce cas, le support de $[S]$ contient une infinité de composantes analytiques irréductibles. L'espace affine $\mathbb{C}^{2}$ étant de Stein, la solution est unique. Mais dans le cas de $P_{2}(\mathbb{C})$ il peut exister une infinité de solutions au problème du bord pour une courbe de classe $A_{1}$ :

Exemple 4. - Du fait que $\mathbb{C}^{2} \subset P_{2}(\mathbb{C})$, nous pouvons considérer l'exemple précédent dans $P_{2}(\mathbb{C})$. Pour tout $i \in \mathbb{N}^{*}$, notons $P_{1}^{i}(\mathbb{C})$ l'unique sphère de Riemann contenant $D_{i}^{+}$et $D_{i}^{-}=P_{1}^{i} \backslash D_{i}^{+}$. Alors, pour tout $i,\left[C_{i}\right]=d\left[D_{i}^{+}\right]$mais on a aussi $\left[C_{i}\right]=d\left(-\left[D_{i}^{-}\right]\right)$. Dans $P_{2}(\mathbb{C}),[\gamma]$ admet donc une infinité de solutions au problème du bord. En effet, toute chaîne de la forme $\sum_{i=1}^{\infty} \pm\left[D_{i}^{ \pm}\right]$où ' \pm ' vaut ' + ' sauf pour un nombre fini d'entiers $i \in \mathbb{N}^{*}$ pour lesquels ' \pm ' vaut '-' est une solution au problème du bord pour $[\gamma]$. De plus, pour tout $i \in \mathbb{N}^{*}$, $P_{1}^{i}$ est une courbe compacte contenant une composante irréductible du support de toute solution au problème du bord pour $[\gamma]$.

Cependant, si l'on se restreint à l'ensemble des solutions au problème du bord à une courbe donnée dont le volume du support est «minimal » (ou «proche de l'être »), alors il existe un ensemble fini de courbes $L_{1}, \ldots, L_{k}$ compactes tel que deux solutions quelconques parmis elles diffèrent d'une chaîne holomorphe combinaison des $L_{1}, \ldots, L_{k}$ (voir la preuve de la proposition 5.15 pour une illustration de ce fait).

\section{Applications et corollaires}

4.1. Problème du bord dans l'espace projectif complexe. - Dans cette section, nous montrons (de manière abrégée) comment appliquer notre théorème principal (ou sa démonstration) pour obtenir de nouvelles preuves des principaux résultats sur le problème du bord dans l'espace projectif. 
Corollaire 4.1 (voir [18], [12], [8]). — Soit $[\Gamma]$ un courant rectifiable, fermé, maximalement complexe de $P_{n}(\mathbb{C}) \backslash P_{(n-p+1)}(\mathbb{C})(p \geq 2)$ dont le support est un compact de classe $A_{2 p-1}$. Alors il existe une $p$-chaîne holomorphe $[T]$ de $P_{n}(\mathbb{C}) \backslash\left(P_{(n-p+1)}(\mathbb{C}) \cup \Gamma\right)$, de masse finie dans $P_{n}(\mathbb{C}) \backslash P_{(n-p+1)}(\mathbb{C})$ telle que $[\Gamma]=d[T]$.

Démonstration. - Pour $p=2, \Gamma \subset P_{n}(\mathbb{C}) \backslash P_{n-1}(\mathbb{C}) \simeq \mathbb{C}^{n}$. Le problème du bord pour $[\Gamma]$ admet donc une solution (voir [8]). Pour $p>2$, soit $H$ un $n-p+1$ espace affine tel que $\Gamma \subset P_{n}(\mathbb{C}) \backslash H$. D'après la propriété de tranchage des courants rectifiables de support de classe $A_{2 p-1}$ (prop. 3.2), quitte à perturber légérement $H$, on peut supposer sans perte de généralité qu'il existe une variété $V \subset G(n-p+2, n)$ formée de $(n-p+2)$-plans contenant $H$ telle que pour presque tout $(n-p+2)$-plan $G_{\nu}$ avec $\nu \in V,\left[\Gamma \cap G_{\nu}\right]$ est un 3-courant rectifiable, fermé et maximalement complexe dans $G_{\nu} \backslash H \simeq \mathbb{C}^{n-p+2}$ et dont le support est de classe $A_{3}$. Le problème du bord pour le courant d'intersection $\left[\Gamma \cap G_{\nu}\right]$ admet donc une solution dans $G_{\nu} \backslash H$. Soit $\Psi: V \times \mathbb{C}^{n-p+2} \rightarrow P_{n}(\mathbb{C}) \backslash H$ une application holomorphe injective identifiant $\{\nu\} \times \mathbb{C}^{n-p+2}$ avec $G_{\nu} \backslash H$ (on a supposé ici que $V$ est assez petit pour avoir l'existence de $\Psi)$. Soit $[\widetilde{\Gamma}]=\Psi^{*}([\Gamma])$ l'image inverse de $[\Gamma]$ par $\Psi$. D'après le théorème 3.1 , le problème du bord est résoluble pour $[\widetilde{\Gamma}]$ dans $V \times \mathbb{C}^{n-p+2}$ (quitte à avoir ici aussi choisi $H$ convenablement pour que les hypothèses du théorème soient vérifiées). Donc, il existe un $(n-p+2)$-plan $P$ tel que le problème du bord pour $[\Gamma]$ admette une solution $[T]$ au voisinage de $P$. Soit $P_{\epsilon}$ un $\epsilon$-voisinage de $P$ dans $P_{n}(\mathbb{C})$. Pour $\epsilon$ assez petit la restriction de $[T]$ à $P_{\epsilon}$ est une $p$-chaîne holomorphe $\left[T_{\epsilon}\right]$ dont le bord est un courant rectifiable maximalement complexe dont le support est de classe $A_{2 p-1}$ et qui coïncide avec [ $\left.\Gamma\right]$ au voisinage de $P$. La résolution du problème du bord pour $[\Gamma]$ et alors équivalente à la résolution du problème du bord pour $[\Gamma]-d\left[T_{\epsilon}\right]$ dont le support est dans $P_{n}(\mathbb{C}) \backslash P$. On est donc ramené au cas où $\Gamma \subset P_{n}(\mathbb{C}) \backslash P_{n-p+2}(\mathbb{C})$. Si $p=3$, le résultat est prouvé. Sinon, en faisant à nouveau la même manipulation, on est ramené au cas $\Gamma \subset P_{n}(\mathbb{C}) \backslash P_{n-p+3}(\mathbb{C})$. Puis par récurrence, on se ramène au cas $\Gamma \subset P_{n}(\mathbb{C}) \backslash P_{n-1}(\mathbb{C}) \simeq \mathbb{C}^{n}$ ce qui montre le résultat.

DÉfinition 4.2 (voir [10]). — Soit $U$ un ouvert de la variété grassmanienne $G(n-p+1, n)$. Un sous-ensemble $K \subset U$ est dit projectivement $k$-générique si pour tout ensemble non $k$-générique $S \subset P_{n}(\mathbb{C})$, l'ensemble $K \backslash G_{S}$ est $k$ générique où

$$
G_{S}=\left\{\nu \in G(n-p+1, n) ; P_{n-p+1}^{\nu}(\mathbb{C}) \cap S \neq \varnothing\right\} .
$$

Une propriété principale des ensembles projectivement $k$-génériques est que si $V \subset U$ est une sous-variété complexe de dimension $p-1$ qui est $(p-2)$ générique, alors il existe $\nu \in V$ tel que $P_{n-p+1}^{\nu} \cap S=\varnothing$, où

$$
S=\left\{z \in P_{n}(\mathbb{C}) ; \operatorname{dim}\left\{\nu \in V ; z \in P_{n-p+1}^{\nu}\right\} \geq 1\right\} .
$$

TOME $130-2002-\mathrm{N}^{\mathrm{O}} 2$ 
Corollaire 4.3 (voir [12], [10]). - Soit $X$ un ouvert $(n-p+1)$-concave de $P_{n}(\mathbb{C})$ avec

$$
X^{*}=\left\{\nu \in G(n-p+1, n) ; P_{n-p+1}^{\nu} \subset X\right\}
$$

connexe. Soit $[\Gamma]$ une combinaison linéaire, localement finie, à coefficients entiers de courants d'intégration sur des sous-variétés réelles $\Gamma_{i}$, orientées, de classe $C^{1}$, de dimension $(2 p-1)$ et maximalement complexe dans $X$. Soit $K$ un sous-ensemble projectivement $(p-2)$-générique de $X^{*}$. Supposons que pour tout $\nu \in K$ :

1) $P_{n-p+1}^{\nu}$ intersecte $\Gamma_{i}$ transversalement en tout point;

2) il existe une 1-chaîne holomorphe $\left[S_{\nu}\right]$ de masse finie de $P_{n-p+1}^{\nu} \backslash \Gamma$ telle que $d\left[S_{\nu}\right]=\left[\gamma_{\nu}\right]$ au sens des courants dans $P_{n-p+1}^{\nu}$ (où $\left[\gamma_{\nu}\right]=\left[\Gamma \cap P_{n-p+1}^{\nu}\right]$ est le courant d'intersection de $P_{n-p+1}^{\nu}$ avec $\left.[\Gamma]\right)$.

Alors il existe une p-chaîne holomorphe $[T]$ de $X \backslash \Gamma$, de masse localement finie, vérifiant $d[T]=[\Gamma]$ dans $X$.

Démonstration. - De la même manière que dans la démonstration de l'implication 1$) \Rightarrow 2$ ), on montre qu'il existe $\nu_{0} \in K$, un voisinage de Stein $V_{0}$ de $S_{\nu_{0}} \cup \gamma_{\nu_{0}}$ (où $\gamma_{\nu_{0}}=\Gamma \cap P_{n-p+1}^{\nu_{0}}$ ) et un sous-ensemble dénombrablement $(n-1)$ générique $K_{0}$ de $K$ tel que pour tout $\nu \in K_{0}, S_{\nu} \subset V_{0}$. De la proposition 5.1, on déduit alors qu'il existe une sous-variété analytique $\widetilde{K} \subset X^{*}$ de dimension $p-1$ et projectivement $(p-2)$-générique tel que pour tout $\nu \in \widetilde{K}$, le problème du bord pour $\left[\gamma_{\nu}\right]$ soit résoluble dans $V_{0}$. Soit $\Psi: \widetilde{K} \times P_{n-p+1}(\mathbb{C}) \rightarrow P_{n}(\mathbb{C})$ la projection canonique qui identifie $\{\nu\} \times P_{n-p+1}(\mathbb{C})$ avec $P_{n-p+1}^{\nu}$. Alors il existe $\nu \in \widetilde{K}$ tel que $\Psi^{-1}(\{z\})$ soit fini pour tout $z \in P_{n-p+1}^{\nu}$. Il existe donc un voisinage $V$ de $\nu \in \widetilde{K}$ tel que la restriction de $\Psi$ à $V \times P_{n-p+1}(\mathbb{C})$ soit propre et d'ordre fini. L'image inverse $[\widetilde{\Gamma}]=\Psi^{*}([\Gamma])$ est donc bien définie et est encore un courant rectifiable, fermé, maximalement complexe. De plus, cette $p$-chaîne a un support de classe $A_{2 p-1}$. D'après le théorème 3.1, il existe un ouvert $U$ de $V$ tel que le problème du bord pour $[\widetilde{\Gamma}]$ admette une solution $[\widetilde{T}]$ dans $U \times P_{n-p+1}(\mathbb{C})$. Mais alors, l'image directe de $[\widetilde{T}]$ donne une solution $[T]=\Psi_{*}([\widetilde{T}])$ au problème du bord pour $[\Gamma]$ dans un voisinage d'un $(n-p+1)$-plan $P$ de $P_{n}(\mathbb{C})$. Soit $P_{\epsilon}$ un $\epsilon$-voisinage de $P$ dans $P_{n}(\mathbb{C})$. Pour $\epsilon$ assez petit, la restriction $\left[T_{\epsilon}\right]$ de $[T]$ à $P_{\epsilon}$ est une $p$-chaîne holomorphe dont le bord est un courant rectifiable maximalement complexe dont le support est de classe $A_{2 p-1}$ et qui coïncide avec $[\Gamma]$ au voisinage de $P$. Le courant $[\Gamma]-d\left[T_{\epsilon}\right]$ a donc un support dans $P_{n}(\mathbb{C}) \backslash P$ et admet une solution $[R]$ au problème du bord d'après le corollaire précédent. La $p$-chaîne holomorphe $[R]+\left[T_{\epsilon}\right]$ nous donne alors une solution au problème du bord pour $[\Gamma]$. 
4.2. Théorème de Hartogs-Levi généralisé. - On note $\Delta$ le disque unité de $\mathbb{C}$,

$$
C(r)=\{z \in \mathbb{C} ;|z|=r\} \quad \text { et } \quad C\left(r_{1}, r_{2}\right)=\left\{z \in \mathbb{C} ; r_{1}<|z|<r_{2}\right\} .
$$

Corollaire 4.4 (théorème de Hartogs-Levi généralisé)

Soit $X$ une variété kählérienne disque convexe. Soit $f$ une application méromorphe, à valeurs dans $X$ et définie sur $C(1-\epsilon, 1) \times \Delta$. Soit $\left\{\ell_{\nu}\right\}_{\nu \in V}$ une famille non dénombrable de droites complexes. On suppose de plus que pour tout $\nu \in V, \ell_{\nu} \cap C(1-\epsilon, 1) \times \Delta \neq \varnothing, \ell_{\nu} \cap C(1-\epsilon, 1) \times b \Delta=\varnothing$ et pour tous $\nu_{1}$, $\nu_{2}, \nu_{3} \in V$ deux à deux différents, $\ell_{\nu_{1}} \cap \ell_{\nu_{2}} \cap \ell_{\nu_{3}} \cap \Delta^{2}=\varnothing$. Supposons que $f$ se prolonge méromorphiquement à $\ell_{\nu} \cap \Delta^{2}$ pour tout $\nu \in V$. Alors $f$ se prolonge méromorphiquement à $\Delta^{2}$.

Démonstration. - L'application $f$ étant méromorphe, l'ensemble des points d'indétermination de $f$ est discret dans $C(1-\epsilon, 1) \times \Delta$. Soir $r \in] 1-\epsilon, 1[$, tel que $M_{r}=C(r) \times \Delta$ ne rencontre aucun point d'indétermination de $f$. La restriction de $f$ à $M_{r}$ est donc lisse et pour tout $\nu \in V \subset G(2,3)$, la droite $\ell_{\nu}$ est transverse à $M_{r}$. Soit

$$
\Gamma_{f}=\left\{(w, c) \in \Delta^{2} \times X ; w \in M_{r}, c=f(w)\right\}
$$

le graphe de $f$. De la même manière que dans la preuve du théorème 2.3, il existe une surface de Riemann $\widetilde{V} \subset G(2,3)$, un point $\nu_{0} \in \widetilde{V}$, un voisinage de Stein $W$ de $S_{\nu_{0}} \cup \gamma_{\nu_{0}}$ (où $S_{\nu_{0}}$ est le graphe de l'extension méromorphe de $f$ à $\ell_{\nu_{0}}$ et $\left.\gamma_{\nu_{0}}=\Gamma_{f} \cap\left(\ell_{\nu_{0}} \times X\right)\right)$, tels que l'ensemble des points $\nu \in \widetilde{V}$ vérifiant $S_{\nu} \subset W$ soit 0-générique. Soit $\phi: W \rightarrow \mathbb{C}^{m}$ un plongement de $W$ dans l'espace affine. D'après l'hypothèse faite sur les droite $\ell_{\nu}$ et le théorème de Levi, $\phi \circ f$ admet une extension holomorphe au voisinage de $\Delta^{2} \cap \ell_{\nu_{0}}$. Et donc $f=\phi^{-1} \circ \phi \circ f$ admet une extension holomorphe au voisinage de $\Delta^{2} \cap \ell_{\nu_{0}}$. L'extension méromorphe à tout $\Delta^{2}$ se fait alors soit en appliquant à nouveau le théorème 2.3 , soit grâce au résultat de [22].

4.3. Généralisations du théorème de Hartogs-Bochner. - Soit $f$ une fonction de classe $C^{1}$ définie sur une variété maximalement complexe $\Gamma$. Alors $f$ est une fonction CR si et seulement si son graphe est maximalement complexe. Dans ce paragraphe, en appliquant le problème du bord pour le graphe de fonctions CR dans différentes situations, nous obtenons une extension analytique de ce graphe et par conséquent une extension analytique de ces fonctions CR.

Corollaire 4.5. - Soient $X$ une variété kählérienne disque convexe et $\Gamma$ une sous-variété réelle, orientée, compacte, de classe $C^{2}$, de dimension $2 p-1$ $(p \geq 2)$, connexe et maximalement complexe de $\mathbb{C}^{n}$. D'après [18], il existe un unique sous-ensemble analytique irréductible $A$ de dimension $p$ et de volume fini de $\mathbb{C}^{n} \backslash \Gamma$ solution au problème du bord pour $[\Gamma]$ (i.e. $d[A]= \pm[\Gamma]$ selon

TOME $130-2002-\mathrm{N}^{\mathrm{O}} 2$ 
l'orientation de $\Gamma)$. Alors toute application $C R$ continue $f: \Gamma \rightarrow X$ admet une extension méromorphe à $A$.

Démonstration. - D'après [24], $\Gamma$ est une variété CR globalement minimale (i.e deux points de $\Gamma$ peuvent toujours être joints par une réunion finie de courbes CR de $\Gamma$ ). Par conséquent, d'après le théorème de Trépreau [38], $\Gamma$ admet une extension en variété complexe $\mathcal{W}$ qui, en chaque point de $\Gamma$, est un voisinage ou un demi-voisinage de ce point. Nous noterons $\mathcal{W}$ cet ensemble analytique. De plus, encore d'après le théorème de Trépreau, $\mathcal{W}$ peut être choisit assez petit pour que $f$ admette une extension holomorphe à $\mathcal{W}$. Soit $\Pi: \mathbb{C}^{n} \rightarrow$ $\mathbb{C}^{p-1}$ la projection sur les $(p-1)$ premières coordonnées de $\mathbb{C}^{n}$. Quitte à changer de coordonnées, on peut toujours supposer que pour tout $z \in \mathbb{C}^{p-1}, \Pi^{-1}(\{z\}) \cap$ $\mathcal{W}$ est une surface de Riemann (pouvant être vide). Quitte à déformer $\Gamma$ dans $\mathcal{W}$, on peut supposer sans perte de généralité que $f$ est lisse sur $\Gamma$ et d'après la proposition 2.4 que

$$
\Gamma_{f}=\left\{z \times(w, y) \in \mathbb{C}^{p-1} \times\left(\mathbb{C}^{n-p+1} \times X\right) ;(z, w) \in \Gamma, y=f(z)\right\}
$$

vérifie les hypothèses $a), b$ ) et $c$ ) du théorème 2.3 avec $U=\mathbb{C}^{p-1}$ et $\omega=$ $\mathbb{C}^{n-p+1} \times X$. La variété $\Gamma$ étant compacte, il existe $R>0$ tel que si $|x|>R$, on ait $\pi^{-1}(x) \cap \Gamma=\varnothing$ (rappelons que $\pi: U \times \omega \rightarrow U$ est la projection sur le premier membre). Le problème du bord pour $\left[\Gamma_{f}\right]$ est donc résoluble dans l'ouvert $\{|x|>R\} \times\left(\mathbb{C}^{n-p+1} \times X\right)$ (avec le courant nul comme solution au problème du bord). La variété $\omega=\mathbb{C}^{n-p+1} \times X$ est bien sûr disque convexe, le théorème 2.3 s'applique donc. Il reste à vérifier que l'extension ainsi obtenue donne une solution globale au problème du bord pour $\left[\Gamma_{f}\right]$. D'après le lemme 2.8 , il existe un ouvert connexe maximal $U \subset \mathbb{C}^{p-1}$ contenant l'ouvert $\{|x|>R\}$ tel que le problème du bord pour $\left[\Gamma_{f}\right]$ admette une solution $\left[T_{U}\right]$ dans $U \times\left(\mathbb{C}^{n-p+1} \times X\right)$ qui est nulle pour $\{|x|>R\}$.

LEMME 4.6. - On peux toujours supposer que $\left[T_{U}\right]$ vérifie la propriété suivante : il n'existe pas d'ouvert $W \subset U$ tel que $T_{U} \cap \pi^{-1}(W)$ contienne un sousensemble analytique de $\pi^{-1}(U)$ de dimension $p$, fermé et non vide.

Démonstration. - Pour cela, il suffit de montrer si $R$ est une composante analytique irréductible de $T_{U}$ fermée dans $\pi^{-1}(W)$ (où $W$ est un ouvert de $U$ ) alors $R$ est fermée dans $\pi^{-1}(U)$. Notons $[R]$ la restriction de $\left[T_{U}\right]$ à $R$. Par définition de $\left[T_{U}\right]$, pour tout point $w \in W, R_{w}=R \cap \pi^{-1}(w)$ est une courbe compacte. En particulier, si on note $\Pi_{2}: \mathbb{C}^{n} \times X \rightarrow \mathbb{C}^{n}$ la projection sur le premier membre, pour tout point $w \in W, \Pi_{2}\left(R_{w}\right)$ est fini (car c'est un sous-ensemble analytique compact de $\left.\mathbb{C}^{n}\right)$. Par conséquent $\Pi_{2 *}([R])$ est une chaîne holomorphe de $\mathbb{C}^{n}$ de dimension inférieure à $p-1$. Or, on a

$$
d \Pi_{2 *}\left(\left[T_{U}\right]\right)=\Pi_{2 *}\left(d\left[T_{U}\right]\right)=\Pi_{2 *}\left(\left[\Gamma_{f}\right]\right)=[\Gamma] .
$$


Par conséquent, le bord (au sens des courants) de $[R]$ ne peut être le courant d'intégration sur une composante de $\Gamma_{f}$ et $[R]$ est donc une $p$-chaîne holomorphe fermée de $U \times \mathbb{C}^{n-p+1} \times X$. Quitte à remplacer $\left[T_{U}\right]$ par $\left[T_{U}\right]-[R]$, on peut donc toujours supposer que $\left[T_{U}\right]$ vérifie la propriété du lemme.

LEMME 4.7. - Le bord $G=\partial U$ est de mesure de Hausdorff $(2 p-3)$-dimensionnelle nulle.

Démonstration. - Notons $W_{\max }$ l'ensemble des point $z \in \mathbb{C}^{p-1}$ ayant un voisinage $W_{z}$ tel que le problème du bord pour $\Gamma_{f}$ admette une solution dans $W_{z} \times\left(\mathbb{C}^{n-p+1} \times X\right)$. Supposons que $G$ est de mesure de Hausdorff $(2 p-3)$ dimensionnelle non nulle. D'après le lemme 2.8 , il existe alors un point $z$ dans $W_{\max } \cap G$. Soit $W_{z}$ un voisinage de $z$ dans $\mathbb{C}^{p-1}$ tel que le problème du bord pour $\left[\Gamma_{f}\right]$ admette une solution $\left[T_{z}\right]$ dans $W_{z} \times \mathbb{C}^{n-p+1} \times X$ (on peut toujours supposer que l'adhérence de $T_{z}$ ne contient aucun sous-ensemble analytique fermé de dimension $p$ de $\left.W_{z} \times \mathbb{C}^{n-p+1} \times X\right)$. Comme $W_{z}$ n'est pas inclus dans $U$, il existe au moins une composante connexe $W$ de $U \cap W_{z}$ tel que les restrictions de $\left[T_{z}\right]$ et de $\left[T_{U}\right]$ à $W \times \mathbb{C}^{n-p+1} \times X$ ne soient pas égales. Mais alors le courant $[R]=\left[T_{z}\right]-\left[T_{U}\right]$ défini dans $W \times\left(\mathbb{C}^{n-p+1} \times X\right)$ est fermé et est donc une $p$-chaîne holomorphe de $W \times\left(\mathbb{C}^{n-p+1} \times X\right)$. Comme les chaînes $\left[T_{U}\right]$ et $\left[T_{z}\right]$ n'ont pas de composantes fermées dans $\pi^{-1}(W)$ (voir lemme précédent), toutes leurs composantes admettent des composantes de $\Gamma_{f}$ dans leur adhérence. Par conséquent, il existe un point $z \in W$ tel que $R \cap \gamma_{z}$ (où $\gamma_{z}=\Gamma_{f} \cap \pi^{-1}(z)$ ) est une courbe lisse non vide (donc n'est pas un ensemble fini). Or, $R \cap \pi^{-1}(z)$ est une courbe compacte et donc sa projection sur $\mathbb{C}^{n}$ est un nombre fini de points. Or cette projection doit contenir au moins la projection d'une composante de la courbe $\gamma_{z}$ ce qui donne la contradiction recherchée.

Le bord $G=\partial U$ ne peut donc déconnecter $\mathbb{C}^{p-1}$ et donc $G=\mathbb{C}^{p-1} \backslash U$. D'après la proposition 5.17 , la solution $[T]$ au problème du bord pour $\left[\Gamma_{f}\right]$ est de volume borné au voisinage de $G$ et admet une extension simple (que l'on notera aussi $[T])$ sur $\left(\mathbb{C}^{p-1} \times \mathbb{C}^{n-p+1} \times X\right) \backslash \Gamma_{f}$ qui est solution au problème du bord pour $\left[\Gamma_{f}\right]$ dans $\mathbb{C}^{p-1} \times \mathbb{C}^{n-p+1} \times X$. Soit

$$
\Pi: \mathbb{C}^{p-1} \times \mathbb{C}^{n-p+1} \times X \longrightarrow \mathbb{C}^{n}
$$

la projection canonique sur les deux premiers membres. On a alors :

$$
d \Pi_{*}([T])=\Pi_{*}(d[T])=\Pi_{*}\left(\left[\Gamma_{f}\right]\right)=[\Gamma]= \pm d[A] .
$$

Par unicité du problème du bord dans $\mathbb{C}^{n}$, on a alors $\Pi_{*}([T])= \pm[A]$. Les $p$ chaînes holomorphes $[T]$ et $[A]$ étant de multiplicité $\pm 1, \Pi_{\mid T}$ est une injection (en dehors d'un sous-ensemble analytique propre de $T$ ). L'application inverse de $\Pi_{\mid T}$ est donc une application méromorphe dont la valeur au bord (au sens des courants) est égale à $\left[\Gamma_{f}\right]$, c'est l'extension méromorphe de $f$.

TOME $130-2002-\mathrm{N}^{\mathrm{O}} 2$ 
De la même manière que dans [33], proposition 6, en remplaçant l'utilisation du théorème d'extension CR-méromorphe par le corollaire précédent, nous obtenons les deux corollaires suivants :

COROllaire 4.8. - Soient $\omega$ une variété kählérienne disque convexe et $\Gamma \subset \omega$ une sous-variété réelle de classe $C^{2}$, de dimension $2 p-1(p \geq 2)$, orientée, connexe, compacte et maximalement complexe. Supposons que $\Gamma$ admette un plongement $C R \phi$ de classe $C^{2}$ dans $\mathbb{C}^{n}$. Alors il existe un sous-ensemble analytique irréductible $A$ de $X \backslash \Gamma$, de dimension $p$, borné et de volume fini dans $\omega$ solution au problème du bord pour $[\Gamma]$ (i.e. $d[A]= \pm[\Gamma]$ selon l'orientation de $\Gamma)$. De plus, si $X$ est une variété kählérienne disque convexe, alors toute application $C R$ continue sur $\Gamma$ et à valeurs dans $X$ admet une extension méromorphe $\grave{a} A$.

Corollaire 4.9. - Soit $M$ une hypersurface de classe $C^{2}$ de $P_{n}(\mathbb{C})(n \geq 2)$, le séparant en deux domaines disjoints $\Omega_{1}$ et $\Omega_{2}$. Supposons qu'il existe une fonction holomorphe non constante définie dans un voisinage connexe de $M$. Alors pour toute variété kählérienne disque convexe $X$, les applications $C R$ continues sur $M$ et à valeurs dans $X$ admettent une extension méromorphe soit $\grave{a} \Omega_{1}$ soit à $\Omega_{2}$. Le choix de $\Omega_{1}$ ou de $\Omega_{2}$ ne dépend pas de l'application CR.

Dans le théorème 2.3, l'obstacle à l'existence d'une solution globale au problème du bord est l'apparition de courbes compactes au voisinage desquelles il n'est plus possible d'appliquer la proposition 5.8. Cependant, si l'on suppose que la variété kählérienne $\omega$, ne contient pas de courbe compacte, il n'y a alors plus d'obstacle à ce que la solution obtenue soit une solution globale et nous obtenons alors le corollaire suivant :

COROllaire 4.10. - Supposons que $\Omega$ est une surface kählérienne disque convexe ne contenant aucune surface de Riemann compacte. Soit $M$ une hypersurface réelle lisse, connexe et compacte de $\Omega$ la séparant en deux composantes connexes $\Omega_{1}$ et $\Omega_{2}$. Alors toute fonction holomorphe $f$ au voisinage de $M$ admet une extension holomorphe sur $\Omega_{1}$ ou sur $\Omega_{2}$. Supposons de plus qu'il existe une fonction holomorphe non constante sur un voisinage connexe de $M$ et que $X$ est une variété kählérienne disque convexe. Alors les applications méromorphes dans un voisinage connexe de $M$ et à valeurs dans $X$ admettent une extension méromorphe à $\Omega_{1}$ ou à $\Omega_{2}$. Le choix de $\Omega_{1}$ ou de $\Omega_{2}$ ne dépend pas de l'application $C R$.

Démonstration. - Soit $f$ une fonction holomorphe non constante définie au voisinage de $M$ (si les fonctions holomorphes au voisinage de $M$ sont constantes, il n'y a rien à montrer). De même que précédemment, en considérant le graphe $\left[\Gamma_{f}\right]$ de la restriction de $f$ sur $M$ (ou une déformation de $M$ ), on montre qu'il existe un ouvert maximal $U_{\max }$ tel que le problème du bord pour $\left[\Gamma_{f}\right]$ admette une solution $[T]$ dans $U_{\max } \times \Omega$. Montrons que $U_{\max }=\mathbb{C}$. D'après le lemme 2.8, 
si $G=\mathbb{C} \backslash U_{\max }$ est de mesure de Hausdorff 1-dimensionnelle non nulle, il existe un point $z \in \mathbb{C}$ tel que $\left[\Gamma_{f}\right]$ admette deux solutions $\left[T_{1}\right]$ et $\left[T_{2}\right]$ distinctes au problème du bord dans un voisinage $W$ de $\{z\} \times X$. Mais alors $[R]=\left[T_{1}\right]-\left[T_{2}\right]$ est une 2-chaîne holomorphe fermée et non nulle dans $W$. En particulier, il existe $x \in W$ tel que $R \cap\{x\} \times \Omega$ (où $R$ est le support de $R$ ) est une courbe compacte non vide de $\{x\} \times \Omega$ ce qui contredit le fait que $\Omega$ ne contient pas de surfaces de Riemann compactes. Par conséquent $G$ est de mesure de Hausdorff 1-dimensionnelle nulle et ne peut déconnecter $\mathbb{C}$. Par conséquent, $G=\mathbb{C} \backslash U_{\max }$. Mais d'après la proposition $5.17,[T]$ est de masse finie au voisinage de $G \times \Omega$ et admet une une extension simple fermée (que l'on notera encore $[T]$ ) qui est solution au problème du bord pour $\left[\Gamma_{f}\right]$ dans $\mathbb{C} \times \Omega$ et montre que $U_{\max }=\mathbb{C}$.

Il reste à montrer que cette solution au problème du bord est bien le graphe d'une extension analytique de $f$. Soit $\Pi: \mathbb{C} \times \Omega \rightarrow \Omega$ la projection canonique sur le second membre, alors $\Pi_{*}([T])$ définie une 2-chaîne holomorphe de $\Omega \backslash M$ et donc

$$
\Pi_{*}([T])=k_{1}\left[\Omega_{1}\right]+k_{2}\left[\Omega_{2}\right]
$$

avec $k_{1}, k_{2} \in \mathbb{Z}$ (cela signifie que $T$ définit un revêtement à $k_{1}$ feuillets au dessus de $\Omega_{1}$ et un revêtement à $k_{2}$ feuillets au dessus de $\Omega_{2}$ ). De plus on a :

$$
\begin{aligned}
d \Pi_{*}([T]) & =\Pi_{*}(d[T])=\Pi_{*}\left(\left[\Gamma_{f}\right]\right)=[M] \\
& =d\left(k_{1}\left[\Omega_{1}\right]-k_{2}\left[\Omega_{2}\right]\right)=k_{1}[M]-k_{2}[M]=\left(k_{1}-k_{2}\right)[M]
\end{aligned}
$$

(on a supposé ici que $M$ est le bord orienté de $\Omega_{1}$ ) et donc on a

$$
k_{1}-k_{2}=1 \text {. }
$$

Pour conclure, il reste à montrer que $k_{1}$ ou $k_{2}$ est égal à zero. En effet, $[T]$ sera alors un revêtement à un seul feuillet au dessus de l'un des côtés de $M$. Ce sera alors le graphe de l'extension holomorphe recherchée de $f$. Soit $z$ la coordonnée holomorphe de $\mathbb{C}$ et soit

$$
[F]=\Pi_{*}(z[T])
$$

Alors $[F]$ vérifie

$$
\bar{\partial}[F]=\bar{\partial} \Pi_{*}(z[T])=\Pi_{*}(z \bar{\partial}[T])=\Pi_{*}\left(z\left[\Gamma_{f}\right]\right)=f[M] .
$$

Comme $f$ est holomorphe au voisinage de $M$, on montre que

$$
[F]=f_{1}\left[\Omega_{1}\right]+f_{2}\left[\Omega_{2}\right]
$$

où les fonctions $f_{i}$ sont des fonctions holomorphes au voisinage de $\bar{\Omega}_{i}$ et vérifient $f_{\mid M}=f_{1 \mid M}-f_{2 \mid M}$. Comme $f$ est supposée non constante, l'un de ces deux fonctions est non constante. Supposons, par exemple, que $f_{1}$ n'est pas constante et montrons alors que $f$ admet une extension holomorphe à $\Omega_{1}$. Pour cela, considérons

$$
\Gamma_{f_{1}, f}=\left\{(z,(y, w)) \in \mathbb{C} \times(\mathbb{C} \times X) ; w \in M, z=f_{1}(w), y=f(w)\right\}
$$

TOME $130-2002-\mathrm{N}^{\mathrm{O}} 2$ 
le graphe du l'application $\left(f_{1}, f\right)$. De la même manière que précédement, en remarquant que $\mathbb{C} \times X$ ne contient aucune courbe compacte, on montre que $\left[\Gamma_{f_{1}, f}\right]$ admet une solution $\left[T_{f_{1}, f}\right]$ au problème du bord dans $\mathbb{C} \times(\mathbb{C} \times X)$. Notons $\Pi_{2}: \mathbb{C} \times \mathbb{C} \times X \rightarrow \mathbb{C} \times X$ la projection définie par $\Pi_{2}(z, y, w)=(z, w)$. Définissons

$$
\begin{aligned}
& \Gamma_{f_{1}}=\left\{(z, w) \in \mathbb{C} \times X ; w \in M, z=f_{1}(w)\right\}, \\
& T_{f_{1}}=\left\{(z, w) \in \mathbb{C} \times X ; w \in \Omega_{1}, z=f_{1}(w)\right\} .
\end{aligned}
$$

On a bien sûr $d\left[T_{f_{1}}\right]=\left[\Gamma_{f_{1}}\right]$ et $\Pi_{2 *}\left(\left[\Gamma_{f_{1}, f}\right]\right)=\left[\Gamma_{f_{1}}\right]$. Par conséquent,

$$
d \Pi_{2 *}\left(\left[T_{f_{1}, f}\right]\right)=\Pi_{2 *}\left(d\left[T_{f_{1}, f}\right]\right)=\Pi_{2 *}\left(\left[\Gamma_{f_{1}, f}\right]\right)=\left[\Gamma_{f_{1}}\right]
$$

et donc $\Pi_{2 *}\left(\left[T_{f_{1}, f}\right]\right)$ est une solution au problème du bord pour $\left[\Gamma_{f_{1}}\right]$.

Lemme 4.11. - On a $\Pi_{2 *}\left(\left[T_{f_{1}, f}\right]\right)=\left[T_{f_{1}}\right]$. Par conséquent, $\Pi_{2}$ définit un biholomorphisme entre $T_{f_{1}, f}$ et $T_{f_{1}}$.

Démonstration. — Soit $[R]=\left[T_{f_{1}, f}\right]-\left[T_{f_{1}}\right]$, alors $[R]$ est une 2-chaîne holomorphe fermée de $\mathbb{C} \times X$ dont le support est, par construction, relativement compact dans $\mathbb{C} \times X$. Mais alors son support $R$ est un ensemble analytique compact de dimension pure 2 de $\mathbb{C} \times X$. Par conséquent, la projection de $R$ sur $\mathbb{C}$ est un sous-ensemble analytique compact de $\mathbb{C}$ et est donc soit vide, soit fini. Or $f_{1}$ étant supposée non constante, on a nécessairement $R=\varnothing$ et donc $\left[T_{f_{1}, f}\right]=\left[T_{f_{1}}\right]$. Comme $\Gamma_{f_{1}}$ et $\Gamma_{f_{1}, f}$ sont connexes, $\left[T_{f_{1}, f}\right]$ et $\left[T_{f_{1}}\right]$ sont des 2 -chaînes holomorphes irréductibles de multiplicité \pm 1 . Par conséquent $T_{f_{1}, f}$ est un revêtement à un seul feuillet au dessus de $T_{f_{1}}$ ce qui termine la preuve du lemme.

Par définition de $T_{f_{1}}, \pi$ définit un biholomorphisme entre $T_{f_{1}}$ et $\Omega_{1}$. Par conséquent, $\pi \circ \Pi_{2}$ définit un biholomorphisme entre $T_{f_{1}, f}$ et $\Omega_{1}$, ce qui montre que $T_{f_{1}, f}$ est le graphe d'une extension holomorphe de l'application $\left(f_{1}, f\right)$. Par conséquent, $f$ admet une extension holomorphe à $\Omega_{1}$, ce qui termine la preuve de la première assertion du corollaire 4.10.

Dans le cas d'une application $\mathrm{CR} g$ à valeurs dans $X$, la démonstration se fait de la même manière en considérant le graphe de l'application $(f, g)$,

$$
\Gamma_{g, f}=\{(z, y, w) \in \mathbb{C} \times X \times \Omega ; w \in M, z=f(w), y=g(w)\}
$$

et en appliquant le théorème 2.3 avec $U=\mathbb{C}$ et $\omega=X \times \Omega$.

4.4. Plongement des structures CR. - Le but de ce paragraphe est de donner une caractérisation des structures CR strictement pseudoconvexes admettant une solution au problème du bord dans une variété $X$ donnée. Dans le cas où $X$ est de dimension 2 et compacte (et donc $M$ une hypersurface réelle de $X)$, une caractérisation de nature topologique est donnée dans [25]. 
Dans le cas où $X$ est kählérienne disque convexe de dimension quelconque, la caractérisation suivante est valide :

Proposition 4.12. - Soit $M$ une sous-variété orientée, compacte, connexe, de classe $C^{2}$ et maximalement complexe de $X$ vérifiant l'une des trois propriétés suivantes:

1) $M$ est plongeable dans l'espace affine et est de dimension supérieure ou égale à 3 ;

2) $M$ est strictement pseudoconvexe et de dimension 5 ;

3) $M$ est strictement pseudoconvexe, de dimension 3 et est le bord d'une variété complexe abstraite.

Alors $M$ admet une solution au problème du bord. Réciproquement, si $M$ est strictement pseudoconvexe et admet une solution au problème du bord alors $M$ est plongeable dans l'espace affine.

En effet, d'après [27], [6], la deuxième propriété implique automatiquement la première. D'après [27], [21], la troisième propriété implique elle aussi la première. Enfin la première propriété implique que $M$ admet une solution au problème du bord d'après le corollaire 4.7. Réciproquement, supposons que $M$ admet une solution au problème du bord dans $X$, d'après les arguments de [21], $M$ est plongeable dans l'espace affine.

Dans le cas où $M$ est de dimension 3, la question se pose alors de savoir s'il est possible de s'affranchir de l'hypothèse de plongeabilité de $M$ dans l'espace affine. En effet, on ne connaît pas d'exemple de structure CR plongeable dans $X$ mais non plongeable dans $\mathbb{C}^{n}$ (voir [12]). Le corollaire suivant permet alors de montrer que de telles variétés n'admettent aucune fonction CR non constante :

Corollaire 4.13. - Soit $M$ une sous-variété compacte, connexe et strictement pseudoconvexe de dimension 3 de X. Alors l'une des deux propriétés suivantes est vérifiée :

1) les fonctions CR continues (ou les distributions CR) sur $M$ sont constantes;

2) $M$ admet une solution au problème du bord dans $X$ et $M$ est plongeable dans l'espace affine.

Démonstration. - Supposons qu'il existe une fonction CR $f: M \rightarrow \mathbb{C}$ non constante. D'après le théorème d'extension de Lewi, $f$ admet une extension holomorphe à une extension analytique locale du côté pseudoconvexe de $M$. Quitte à déformer $M$ dans cette extension, on peut toujours supposer que $f$ est lisse et que

$$
\Gamma_{f}=\{(f(x), x) \in \mathbb{C} \times X ; x \in M\}
$$

le graphe de $f$ vérifie les hypothèses $a), b$ ) et $c$ ) du théorème 2.3 avec $U=\mathbb{C}$ et $\omega=X$. 
La fonction $f$ étant continue, son module admet un maximum $R$ sur $M$. Le problème $\mathrm{du}$ bord est donc résoluble pour $\left[\Gamma_{f}\right]$ dans l'ouvert $\{|z|>R\} \times X$ (prendre comme solution $[T]=0$ ). D'après le lemme 2.8, il existe un ouvert connexe maximal $U_{\max }$ contenant $\{|z|>R\}$ tel que le problème du bord pour $\left[\Gamma_{f}\right]$ admette une solution dans $U_{\max } \times X$ nulle pour $|z|>R$. Soit

$$
[T]=\sum_{i \in I} n_{i}\left[T_{i}\right]
$$

la décomposition de $[T]$ en composantes irréductibles.

LEMME 4.14. - La 2-chaîne holomorphe $[T]$ est positive (i.e. pour tout $i \in I$, $\left.n_{i} \geq 0\right)$.

Démonstration. - Soit $\pi: \mathbb{C} \times X \rightarrow \mathbb{C}$ la projection canonique sur $\mathbb{C}$. Pour tout $i \in I, \pi\left(T_{i}\right)$ est un ouvert de $\mathbb{C}$. Notons

$$
W=\bigcup_{i \in I, n_{i}<0} \pi\left(T_{i}\right) \quad \text { et } \quad V=U_{\max } \backslash \bar{W}
$$

Supposons par l'absurde que l'ouvert $W$ est non vide et donc que $V$ est un ouvert strictement inclus dans $U_{\max }$. L'ensemble $G=\partial V \cap U_{\max }$ est alors de mesure de Hausdorff 1-dimensionnelle non nulle car $W$ est borné. Il existe donc un point $z \in G$, tel que $\{z\} \times X$ soit transverse à $T$. En particulier, $\left[S_{z}\right]=$ $[T, \pi, z]$ est une 1-chaîne holomorphe positive. Par définition de $V$, il existe $i \in I$ tel que $n_{i}<0$ et $\{z\} \times X$ soit tangent à $\partial T_{i}$ et vérifie $\{z\} \times X \cap T_{i}=\varnothing$. Il existe donc un point $x \in \gamma_{z}$ tel que $\{z\} \times X$ soit tangent en $x$ à $\partial T_{i}$ et tel qu'il existe un voisinage $V_{x}$ de $x$ tel que $d\left[T_{i}\right]= \pm\left[\Gamma_{f}\right]$ dans $V_{x}$. Comme $\{z\} \times X$ est tangent à $\Gamma_{f}$ en $x$ et que $\Gamma_{f}$ est strictement pseudoconvexe, nécessairement $\{z\} \times X$ est tangent du côté concave de $\Gamma_{f}$ et donc $T_{i}$ est du côté convexe de $\Gamma_{f}$ et par conséquent $d\left[T_{i}\right]=\left[\Gamma_{f}\right]$ dans $V_{x}$. Soit $n_{j} \geq 0$ la multiplicité de la composante irréductible $T_{j}$ (si elle existe, sinon poser $n_{j}=0$ ) de $[T]$ dans $U_{\max } \times X$ vérifiant $d\left[T_{j}\right]= \pm\left[\Gamma_{f}\right]$ dans $V_{x}$. Du fait que $\{z\} \times X$ est tangent à $\Gamma_{f}$ en $x, T_{j}$ est nécessairement du côté concave de $\Gamma_{f}$ et par conséquent, on a $d\left[T_{j}\right]=-\left[\Gamma_{f}\right]$ dans $V_{x}$. Comme $\left[\Gamma_{f}\right]$ est le courant d'intégration sur la variété $\Gamma_{f}$, il est de multiplicité 1 en tout point. On a donc $n_{i}-n_{j}=1$ et par conséquent $n_{i}=n_{j}+1>0$ ce qui donne la contradiction recherchée.

LEMME 4.15. - Soit $[\Gamma]$ un $(2 p-1)$-courant rectifiable fermé, maximalement complexe et dont le support est de classe $A_{2 p-1}$ d'une variété complexe $Y$. Soient $\left[T_{1}\right]$ et $\left[T_{2}\right]$ deux p-chaînes holomorphes de $Y \backslash \Gamma$, positives et solutions au problème du bord pour $[\Gamma]$ dans $Y$. Supposons que $T_{1} \cup \Gamma$ et $T_{2} \cup \Gamma$ ne contiennent aucun sous-ensemble analytique de dimension $p$ de $Y$. Alors $\left[T_{1}\right]=\left[T_{2}\right]$. 
Démonstration. - En effet, la $n$-chaîne holomorphe $\left[T_{1}\right]-\left[T_{2}\right]$ est fermée dans $Y$. On a donc

$$
\left[T_{1}\right]-\left[T_{2}\right]=\sum_{i \in I} n_{i}\left[L_{i}\right]
$$

où $n_{i} \in \mathbb{Z}^{*}$ et $L_{i}$ sont des sous-ensemble analytiques de $Y$ de dimension pure $p$. Supposons que $I$ n'est pas vide. Soit donc $i \in I$ et notons $\left[L_{i}^{1}\right]$ et $\left[L_{i}^{2}\right]$ les restrictions de $\left[T_{1}\right]$ et $\left[T_{2}\right]$ à $L_{i}$; on a donc

$$
\left[L_{i}^{1}\right]-\left[L_{i}^{2}\right]=n_{i}\left[L_{i}\right]
$$

Quitte à intervertir le rôle de $\left[L_{1}\right]$ et $\left[L_{2}\right]$, on peut toujours supposer que $n_{i}>0$. On a alors $L_{i} \not \subset L_{i}^{1} \cup \Gamma$ et $L_{i} \not \subset L_{i}^{2} \cup \Gamma$ car $T_{1} \cup \Gamma$ et $T_{2} \cup \Gamma$ ne contiennent pas de sous-ensembles analytiques de dimension $p$ de $Y$. Il existe un composante irréductible $R$ de $L_{i} \backslash \Gamma$ telle que $[R]$ soit de multiplicité 0 dans $\left[L_{1}\right]$. Par hypothèse, on a

$$
k \geq 0
$$

où $k$ est la multiplicité de $[R]$ dans $\left[L_{2}\right]$. On a alors

$$
0-k=n_{i}
$$

et donc $n_{i}$ est négatif ce qui donne la contradiction recherchée.

Soit $G=\partial\left(\mathbb{C} \backslash U_{\max }\right)$. Si $G \neq \varnothing$, alors nécessairement $G$ est de longueur non nulle. En effet, si $G$ était de longueur nulle, d'après le contrôle du volume du lemme 2.7, $[T]$ serait une 2-chaîne holomorphe de $(\mathbb{C} \backslash G) \times X$ de masse finie dans $\mathbb{C} \times X$. Par conséquent, d'après [5], [26], [20], [35], [2], [T] admettrait une extension simple à $((\mathbb{C} \backslash G) \times X) \backslash \Gamma_{f}$ qui serait encore une solution au problème du bord pour $\left[\Gamma_{f}\right]$. Ceci contredit la maximalité de $U_{\max }$. Par conséquent, de la même manière que dans le lemme 2.8 , on montre qu'il existe un sous-ensemble $\widetilde{G} \subset G$ de longueur non nulle tel que pour tout point $z \in \widetilde{G}$, il existe une suite $\left\{w_{i}^{z}\right\}$ de points de $U_{\max }$ pour lesquels les 1-chaînes holomorphes $\left[S_{w_{i}^{z}}\right]=$ $\left[T, \pi, w_{i}^{z}\right]$ (resp. les supports $S_{w_{i}^{z}}$ ) convergent au sens des courants (resp. au sens de Bishop) vers une 1-chaîne holomorphe $\left[S_{z}\right]$ encore solution au problème du bord pour $\left[\gamma_{z}\right]=\left[\Gamma_{f}, \pi, z\right]$ (resp. vers un ensemble analytique $S_{z}$ ). La chaîne holomorphe $[T]$ étant positive, les 1 chaînes holomorphes $\left[S_{w_{i}^{z}}\right]$ le sont aussi. Par conséquent $\left[S_{z}\right]$ l'est aussi et donc $S_{z}=\operatorname{supp}\left[S_{z}\right]$. Soit $\left[\widetilde{S}_{z}\right]$ l'unique 1-chaîne holomorphe positive solution au problème du bord pour $\left[\gamma_{z}\right]$ dont l'adhérence du support ne contient pas de courbe compacte. Alors, de la même manière que dans le théorème principal, on montre qu'il existe un point $z_{0} \in \widetilde{G}$ et un voisinage ouvert et connexe $W_{z_{0}}$ de $z_{0}$ tel que le problème du bord pour $\left[\Gamma_{f}\right]$ admette une solution positive $[\widetilde{T}]$ au problème du bord dans $W_{z_{0}} \times X$. Mais alors, d'après le lemme 4.15, $[T]$ et $[\widetilde{T}]$ sont égales dans $W_{z_{0}} \cap U_{\max }$, ce qui contredit la maximalité de $U_{\max }$. Finalement, $G=\varnothing$ et donc que $\left[\Gamma_{f}\right]$ admet une solution $[T]$ au problème du bord dans $\mathbb{C} \times X$. L'image directe de $[T]$ par

TOME $130-2002-\mathrm{N}^{\mathrm{O}} 2$ 
la projection sur $X$ est une solution au problème du bord pour $[M]$ dans $X$, ce qui termine la preuve du corollaire.

\section{Annexes.}

5.1. Problème du bord non borné dans $\Delta_{n} \times \bar{\Delta}_{m}$. - Notons $\Delta_{q}$ le polydisque unité de $\mathbb{C}^{q}$. Nous nous intéressons ici au problème du bord pour les variétés maximalement complexes de dimension $2 n+1$ de $\Delta_{n} \times \mathbb{C}^{m}$ dont la projection sur $\mathbb{C}^{m}$ est bornée. En général il n'existe pas de solution au problème du bord pour de telles variétés. En effet, il suffit de prendre un courbe fermée $\gamma \subset \Delta_{m}$ qui n'est pas le bord d'une surface de Riemann et de poser $\Gamma=\mathbb{C}^{n} \times \gamma$, pour obtenir un contre-exemple. Il est tout de même possible de donner une condition nécessaire et suffisante. En fait, cette condition est déjà implicitement utilisée dans [8] et nous nous bornons ici à reformuler les propositions de [8] dans ce cadre.

On dit qu'un compact $A \subset \mathbb{R}^{n}$ est géométriquement $m$-rectifiable ou encore est de classe $A_{m}$ si $A$ est $\left(\mathcal{H}_{m}, m\right)$-rectifiable et si le cône tangentiel de $A$ en $\mathcal{H}_{m}$-presque tout point est un espace vectoriel réel de dimension $m$ (où $\mathcal{H}_{m}$ est la mesure de Hausdorff de dimension $m$ ).

Soit $X$ un espace complexe, on appelle p-chaine holomorphe de $X$ toute somme localement finie $[T]=\sum n_{j}\left[V_{j}\right]$ à coefficients $n_{j}$ dans $\mathbb{Z}$ de sousensembles analytiques irréductibles et deux à deux différents $V_{j}$ de dimension $p$ de $X$. On appelle volume de $[T]$, l'expression

$$
\operatorname{Vol}[T]=\sum\left|n_{j}\right| \operatorname{Vol} V_{j}
$$

où $\operatorname{Vol} V_{j}$ est le volume $2 p$-dimensionnel de l'ensemble analytique $V_{j} ; \operatorname{Vol}[T]$ est aussi la masse du courant $[T]$. On notera $T$ le support de la $p$-chaîne holomorphe $[T]$ (i.e. $T=\bigcup_{\left\{j ; n_{j} \neq 0\right\}} V_{j}$ ). Dans la suite, si $[T]$ est un courant on notera aussi $\operatorname{Vol}[T]$ la masse de ce courant.

Soient $X, Y$ deux variétés réelles lisses, $Y$ de dimension $p \leq m$ et $f: X \rightarrow Y$ une application $C^{\infty}$ et $\Gamma$ un courant plat de dimension $m$ (en particulier les courants rectifiables sont plats). Alors pour $\mathcal{H}_{p}$-presque tout $y \in Y$, la tranche $[\Gamma, f, y]$ est un courant plat de dimension $m-p$ de support inclus dans $\Gamma \cap f^{-1}(y)$ vérifiant :

$$
\int_{Y} \Phi(y)([\Gamma, f, y], \Psi) \cdot \Omega=\left[\Gamma, f^{*}(\Phi \cdot \Omega) \wedge \Psi\right]
$$

où $\Psi$ est une $(m-p)$-forme à support compact, $\Phi$ une fonction à support compact et $\Omega$ est la forme volume de $Y$ (voir [14]).

Soit $[\Gamma]$ un courant rectifiable dont le support $\Gamma$ est de classe $A_{2 n+1}$ de $\mathbb{C}^{n+m}$. Alors, pour presque tous les $n$-plan $\mathbb{C}_{\nu}^{n} \subset \mathbb{C}^{n+m}$ et pour presque tous les points 
$z \in \mathbb{C}_{\nu}^{n}$, la tranche $\left[\Gamma, \pi_{\nu}, z\right]$ (où $\pi_{\nu}$ est la projection orthogonale sur $\mathbb{C}_{\nu}^{n}$ ) est un courant rectifiable dont le support est de classe $A_{1}$ (voir [8] Lemme 1.4).

Notons $\pi: \Delta_{n} \times \mathbb{C}^{m} \rightarrow \Delta_{n}$ la projection $\left(z_{1}, \ldots, z_{n}\right) \times\left(w_{1}, \ldots, w_{m}\right) \mapsto$ $\left(z_{1}, \ldots, z_{n}\right)$. Soit $[\Gamma]$ un courant rectifiable, fermé et maximalement complexe de $\Delta_{n} \times \mathbb{C}^{m}$ dont le support $\Gamma$ est de classe $A_{2 n+1}$ et admet une projection bornée sur $\mathbb{C}^{m}$. Pour presque tout $z \in \Delta_{n}$, la tranche $[\Gamma, \pi, z]$ est un courant rectifiable fermé de dimension 1 .

Soient $\chi_{\epsilon_{j}}$ des fonctions de classe $C^{\infty}$, définies sur $\mathbb{C}, \chi_{\epsilon_{j}}(x)=0$ pour $|x|<<\epsilon_{j}$ et $\chi_{\epsilon_{j}}=(1 / 2 \pi) i$ pour $|x|>\epsilon$.

Proposition 5.1 (voir [8]). - Sous les hypothèses ci-dessus, pour tout $z \in \Delta_{n}$, pour toute $(1,0)$-forme holomorphe $\phi$ de $\mathbb{C}^{n}$, la fonction

$$
\mathcal{M}(z, \phi)=\left([\Gamma], \phi \wedge \bigwedge_{j=1}^{n} d \chi_{\epsilon_{j}}\left(\zeta_{j}-z_{j}\right) \wedge \frac{d \zeta_{j}}{\zeta_{j}-z_{j}}\right)
$$

est indépendante des $\chi_{\epsilon_{j}}$ et égale à $([\Gamma, \pi, z], \phi)$ quand cette dernière est bien définie. En particulier la fonction $\mathcal{M}(z, \phi)$ est holomorphe dans $\Delta_{n}$.

Cette proposition montre que la condition des moments (cas où $\phi$ est une (1,0)-forme holomorphe) sur les tranches d'une variété maximalement complexe varie de manière holomorphe. Le principe du prolongement analytique nous dit alors que si la condition des moments est vérifiée pour un sous-ensemble assez grand $K \subset \Delta_{n}$, elle est vérifiée sur tout $\Delta_{n}$. Il est alors possible de recoller ces tranches pour trouver une solution au problème du bord pour $[\Gamma]$.

Proposition 5.2 (voir [8]). - Soit $K$ un sous-ensemble de $\Delta_{n}$, non inclus dans une hypersurface analytique de $\Delta_{n}$. Supposons que pour tout $\nu \in K$, $[\Gamma, \pi, \nu]$ est bien définie et est un 1-courant rectifiable fermé dont le support est de classe $A_{1}$ et que pour toute 2 -forme lisse $\Theta$ sur $\mathbb{C}^{m}$ on ait $[\Gamma] \wedge \Theta=0$. Alors, les propositions suivantes sont équivalentes :

1) $\mathcal{M}(\nu, \phi)$ est nulle pour tout $\nu \in K$ et toute $(1,0)$-forme holomorphe $\phi$ de $\mathbb{C}^{n}$ (i.e. pour tout $\nu \in K,\left[\gamma_{\nu}\right]$ vérifie la condition des moments).

2) Pour tout $\nu \in K$, il existe une 1-chaîne holomorphe $\left[S_{\nu}\right]$ de masse finie de $\Delta_{n} \times \mathbb{C}^{m} \backslash \Gamma$ telle que $d\left[S_{\nu}\right]=[\Gamma, \pi, \nu]$.

3) Il existe une p-chaîne holomorphe $[T]$ de $\Delta_{n} \times \mathbb{C}^{m} \backslash \Gamma$ dont la projection sur $\mathbb{C}^{m}$ est bornée et telle que $d[T]=[\Gamma]$.

Démonstration. - L'équivalence entre 1) et 2) provient directement de [8]. Pour toute $\phi$ fixé, $\mathcal{M}(z, \phi)$ est holomorphe en $z$. L'ensemble des zéros de $\mathcal{M}(z, \phi)$ est donc une hypersurface analytique $H_{\phi}$ de $\Delta_{n}$ contenant $K$. L'intersection $H$ de tous les $H_{\phi}$ est donc un ensemble analytique contenant $K$. Comme $H$ contient $K$ il ne peut être de dimension inférieure ou égale à $n-1$. On a donc $H=\Delta_{n}$. De manière identique à [8], on montre que $\Gamma$ admet une solution $T$ au problème du bord dans $U$. 
Remarque 5.3. - L'hypothèse $[\Gamma] \wedge \Theta=0$ pour toute 2 -forme verticale $\Theta$ implique que pour $\mathcal{H}_{2 n+1}$-presque tout $z \in \Gamma$ tel que la multiplicité de $[\Gamma]$ en $z$ soit non nulle, l'espace tangent à $\Gamma$ en $z$ ne contient pas de droite verticale. En particulier, $[\Gamma]$ ne contient pas de « composantes verticales ».

\subsection{Propriétés de convergence des suites de chaînes holomorphes}

Nous nous intéressons ici à la convergence au sens de Bishop (ou de Hausdorff) des suites de surfaces de Riemann de volumes uniformément bornés :

DÉfinition 5.4. - Soit $\left\{E_{j}\right\}$ une suite de sous-ensembles d'un espace métrique $X$. On dit que la suite $\left\{E_{j}\right\}$ converge au sens de Hausdorff (ou au sens de Bishop si les $E_{j}$ sont des surfaces de Riemann) vers un ensemble $E \subset X$ (on note $\left.E_{j} \rightarrow E\right)$ si :

1) l'ensemble $E$ coïncide avec l'ensemble limite de la suite $\left\{E_{j}\right\}$, i.e. est formé de l'ensemble des points de la forme $\lim _{j_{\nu}} x_{j_{\nu}}, x_{j_{\nu}} \in E_{j_{\nu}}$ (en particulier, $E$ est fermé dans $X)$;

2 ) pour tout compact $K \subset E$ et pour tout $\epsilon>0$, il existe un indice $j(\epsilon, K)$ tel que $K$ appartient au $\epsilon$-voisinage de $E_{j}$ dans $X$ pour tout $j>j(\epsilon, K)$.

Proposition 5.5 (voir [5] et aussi [7]). - Soit $\left\{A_{j}\right\}$ un suite de sousensembles analytiques de dimension pure 1 d'une variété complexe $X$ dont le volume est uniformément borné sur tout compact :

$$
\mathcal{H}_{2}\left(A_{j} \cap K\right) \leq M_{K}<\infty \text { pour tout } K \subset \subset X
$$

et admettant un point d'accumulation dans $X$. Alors il existe une suite extraite $\left\{A_{\phi(j)}\right\}$ de la suite $\left\{A_{j}\right\}$ qui converge dans $X$ vers un sous-ensemble analytique $A$ de dimension pure 1 . De plus, on a l'inégalité

$$
\underset{j \longrightarrow \infty}{\lim _{\longrightarrow}} \mathcal{H}_{2}\left(A_{\phi(j)} \cap U\right) \geq \mathcal{H}_{2}(A \cap U)
$$

pour tout ouvert $U \subset \subset X$.

Dans le cas des chaînes holomorphes, ce théorème se généralise aussi :

Proposition 5.6 (voir $[17])$. - Soit $\left\{\left[A_{j}\right]\right\}$ une suite de 1-chaînes holomorphes d'une variété complexe $X$ dont le volume est uniformément borné sur tout compact. Alors il existe une suite extraite $\left\{\left[A_{\phi(j)}\right]\right\}$ de la suite $\left\{\left[A_{j}\right]\right\}$ convergeant vers une 1-chaîne holomorphe $[A]$ de $X$ au sens des courants.

Proposition 5.7. - Soient $\omega$ une variété complexe munie d'une distance $d$ et $K \subset \omega$ un compact. Soit $V_{\epsilon}=\{z \in \omega, d(z, K) \leq \epsilon\}$ un $\epsilon$-voisinage de $K$. Alors il existe une constante $C_{\epsilon}^{K}$ telle que tout ensemble analytique $A$ de $\omega \backslash K$ irréductible, de dimension 1 dont l'adhérence $\bar{A}$ rencontre $K$ et $\omega \backslash V_{\epsilon}$ vérifie $\operatorname{Vol} A \geq C_{\epsilon}^{K}$. 
Démonstration. - Soit $M=\left\{z \in \omega, d(z, K)=\frac{\epsilon}{2}\right\}$. Le compact $M$ déconnecte $\omega$. Il existe donc un point $w \in M \cap A$. Notons $B\left(w, \frac{1}{2} \epsilon\right)$ la boule de rayon $\frac{1}{2} \epsilon$ et de centre $w$. L'intersection $A \cap B\left(w, \frac{1}{2} \epsilon\right)$ est donc un sous-ensemble analytique fermé et non vide de $B\left(w, \frac{1}{2} \epsilon\right)$. Or pour tout compact $K$ d'une variété complexe et pour tout $\epsilon>0$, il existe une constante $C_{\epsilon}^{K}$ minorant le volume des sous-ensembles analytiques passant par le centre d'une boule de rayon $\frac{1}{2} \epsilon$ qui rencontre $K$ (voir [7] ou la proposition 5.5). D'où le résultat.

5.3. Voisinage de Stein des surfaces de Riemann à bord. - D'après un résultat de Siu [36], toute surface de Riemann n'ayant pas de composante compacte dans un espace complexe $X$ admet un voisinage de Stein. Dans [29], il est montré que si $S$ est une surface de Riemann à bord $\gamma$ connexe et lisse de l'espace projectif et telle que $S \cup \gamma$ ne contient aucune surface de Riemann compacte, alors $S \cup \gamma$ admet lui aussi un voisinage de Stein connexe. Dans [11], ce résultat est étendu au cas où $\gamma$ est une réunion finie, disjointe de courbes de Jordan, de longeur finie et $S$ un sous-ensemble analytique de dimension pure 1 et borné de $X \backslash \gamma$. En fait, la preuve donnée dans [29], [11] est encore valide dans le cas de courbes de classe $A_{1}$ :

Proposition 5.8. - Soit $X$ une variété complexe. Soient $\gamma \subset X$ un compact de classe $A_{1}$ et $S$ un sous-ensemble analytique de dimension 1 de $X \backslash \gamma$, relativement compact dans $X$. Supposons que $S \cup \gamma$ ne contient aucune surface de Riemann compacte de $X$. Alors il existe un ouvert de Stein $V \subset X$ voisinage de $S \cup \gamma$.

En effet, dans [29], [11], le bord de $S$ est supposé lisse uniquement afin de s'assurer que $S$ admet un nombre fini de composantes irréductibles et que l'on peut rendre $\gamma$ connexe en lui ajoutant un nombre fini de courbes lisses. Dans le cas où $\gamma$ est de classe $A_{1}$, comme nous l'avons vu, $S$ peut avoir un nombre infini de composantes irréductibles et il n'est pas toujours possible de rendre $\gamma$ connexe en lui ajoutant une courbe de longeur finie. Cependant, dans notre cas, on n'a pas besoin que le voisinage de Stein obtenu soit connexe (ce qui dispense dans la preuve de rendre $\gamma$ connexe). De plus, si $V$ est un voisinage de $\gamma$, seul un nombre fini des composantes de $S$ ne seront pas incluses dans $V$ et il suffit alors d'appliquer la démonstration de [29], [11] aux composantes non incluses dans $V$ (pour un $V$ bien choisi).

Dans la suite de ce paragraphe, nous adaptons la preuve de [29], [11] au cas où le bord $\gamma$ est de classe $A_{1}$. La preuve repose principalement sur la construction suivante dûe à Mihalache :

Lemme 5.9. - Soit $C$ un compact d'une variété complexe $X$. Soient $U_{1} \subset \subset U_{2}$ deux ouverts de $X$. Notons $A=C \cap \bar{U}_{2}, B=C \backslash U_{1}, \widetilde{A}=A \cap \partial U_{2}$. Supposons de plus que ces ensembles vérifient les deux propriétés suivantes :

TOME $130-2002-\mathrm{N}^{\mathrm{O}} 2$ 
1) B admet une base de voisinages par des ouverts strictement pseudoconvexes à bord lisse et relativement compacts dans $X$;

2) il existe une base $\mathcal{A}$ de voisinages de $\widetilde{A}$ (relativement compact dans $X$ ), telle que si $V_{\tilde{A}} \in \mathcal{A}$, alors $\bar{V}_{\tilde{A}} \cup A$ admet une base de voisinages par des ouverts strictement pseudoconvexes à bord lisse.

Alors $C$ admet un voisinage lisse par morceaux et strictement pseudoconvexe. En particulier, d'après Grauert [15], si C ne contient pas d'ensemble analytique compact de dimension $\geq 1$, il admet un voisinage de Stein.

Démonstration. - Tous les voisinages considérés sont des voisinages d'ensembles compacts. Par conséquent, on peut toujours supposer que tous les voisinages que l'on considérera par la suite admettent un nombre fini de composantes connexes. Soit $V_{B}$ un voisinage strictement pseudoconvexe à bord lisse de $B$ tel que $V_{B} \cap \partial U_{2} \subset V_{\tilde{A}}$. Soit $V_{A}$ un voisinage strictement pseudoconvexe à bord lisse de $\bar{V}_{\tilde{A}} \cup A$ tel que $V_{A} \cap \partial U_{1} \subset \subset V_{B}$ et dont le bord est transverse au bord de $V_{B}$. Soit $W$ l'ouvert défini comme suit :

1) $W \cap U_{1}=V_{A} \cap U_{1}$;

2) $W \cap\left(U_{2} \backslash U_{1}\right)=V_{A} \cap V_{B}$;

3) $W \backslash U_{2}=V_{B} \backslash U_{2}$.

Alors $W$ est bien défini et est lisse par morceaux et strictement pseudoconvexe car il est localement intersection finie de domaines strictement pseudoconvexes à bords lisses.

Lemme 5.10 (voir [11]). — Soient $K$ un compact méromorphiquement convexe dans une variété de Stein $Z$ et $H$ un compact de mesure de Haussdorff 2-dimensionnelle nulle. Alors $K \cup H$ est méromorphiquement convexe dans $Z$.

Lemme 5.11. - Soient $U, V \subset X$ deux ouverts de Stein et $C$ un compact de $Z=U \cup V$ de mesure de Hausdorff 2-dimensionnelle nulle. Alors $C$ admet un voisinage de Stein dans $Z$.

Démonstration. - Soient $U_{1} \subset \subset U_{2} \subset \subset U$ deux ouverts strictement pseudoconvexes, à bord lisse et vérifiant $C \backslash U_{1} \subset V$. Soit $A=C \cap \bar{U}_{2}, B=C \backslash U_{1}$ et $\widetilde{A}=A \cap \partial U_{2}$. D'après le lemme 5.10, comme $\mathcal{H}_{2}(B)=0, B$ admet une base de voisinages méromorphiquement convexes dans $V$. De même, comme $\mathcal{H}_{2}(\widetilde{A})=0, \widetilde{A}$ admet un voisinage $V_{\tilde{A}}$ dont l'adhérence est méromorphiquement convexe dans $U$. Encore d'après le lemme $5.10, \bar{V}_{\tilde{A}} \cup A$ est méromorphiquement convexe dans $U$. Par conséquent, d'après le lemme 5.9, $C$ admet une base de voisinages strictement pseudoconvexes dans $Z=U \cup V$. D'après Grauert, un tel voisinage contient au plus un ensemble analytique compacts maximal de dimension supérieure ou égale à 1 . Par conséquent, en prenant le voisinage strictement pseudoconvexe assez petit, il ne peut contenir de tels ensembles analytiques compacts et sera, par conséquent, de Stein. 
Soit $H$ un compact de mesure de Hausdorff 2-dimensionnelle nulle. En recouvrant $H$ par des ouverts de Stein et en appliquant successivement le lemme précédent, nous obtenons :

Lemme 5.12. - Soit $H \subset X$ un compact de mesure de Hausdorff 2-dimensionnelle nulle. Alors $H$ admet un voisinage de Stein. En particulier, tout compact de classe $A_{1}$ admet une base de voisinages de Stein.

Enfin, nous rappelons que, d'après [28], [8], si $\ell$ est une courbe de classe $A_{1}$ d'une variété de Stein $V$, alors l'enveloppe d'holomorphie $\widehat{\ell}$ de $\ell$ vérifie que $\widehat{\ell} \backslash \ell$ est un sous-ensemble analytique de dimension pure 1 et de volume fini de $V \backslash \ell$.

Preuve de la proposition 5.8. - Soit $W$ un voisinage de Stein de $\gamma$. Notons $S_{1}, \ldots, S_{k}$ les composantes irréductibles de $S$ qui ne sont pas incluses dans $W$. Notons $L_{1}, \ldots, L_{\ell}$ les courbes compactes irréductibles de $X$ contenant l'une des $S_{1}, \ldots, S_{k}$. Comme $S \cup \gamma$ ne contient pas de courbe complexe compacte, choisissons $V \subset W$ un voisinage de Stein de $\gamma$ tel que pour tout $i \in\{1, \ldots, \ell\}$, $L_{i} \not \subset V \cup S$. Soit $\widehat{\gamma}$ l'enveloppe d'holomorphie de $\gamma$ dans $V$. D'après le choix de $V, S \cup \widehat{\gamma}$ ne contient pas de courbe complexe compacte. Soient $[F]$ le courant d'intégration sur $S \cup(\widehat{\gamma} \backslash \gamma)$ et $a$ le support de $d[F]$ (on a alors $a \subset \gamma$ ). Alors, d'après [26], [20], [35], [2], $F=\operatorname{supp}[F]$ est un sous-ensemble analytique de dimension 1 de $X \backslash a$. Quitte à remplacer $S$ par $S \cup F$ et $\gamma$ par $a$, on peut supposer sans perte de généralité que $\gamma=\operatorname{supp} d[S]$ et que $\widehat{\gamma} \backslash \gamma \subset S$ (où $\widehat{\gamma}$ est l'enveloppe d'holomorphie de $\gamma$ dans $V$ ). Soient $\widetilde{S}$ la réunion des composantes de $S$ non incluses dans $V$ et $\widetilde{\gamma}=\operatorname{supp} d[\widetilde{S}]$. Alors $\widetilde{\gamma}$ est une courbe incluse dans $\gamma$ et holomorphiquement convexe dans $V$. L'ensemble $\alpha=\overline{\gamma \backslash \widetilde{\gamma}}$ vérifie alors $\widehat{\alpha} \backslash \alpha=\widehat{\gamma} \backslash \gamma=S \backslash \widetilde{S}$. Soit $V_{2} \subset V$ un ouvert de Stein à bord lisse de $V$ tel que $\widehat{\gamma} \subset \subset V_{2}$ et tel que $\partial V_{2}$, le bord de $V_{2}$, soit lisse et transverse à $S$. L'intersection $\ell=S \cap \partial V_{2}$ est alors une courbe lisse incluse dans $V$ et donc $L=\widehat{\ell} \backslash \ell$ est une réunion finie de sous-ensembles analytiques de dimension pure 1 de $V \backslash \ell$. Soit $V_{3} \subset \subset V_{2}$ un ouvert de Stein à bord $\partial V_{3}$ lisse et transverse à $S$ tel que $\widehat{\gamma} \subset \subset V_{3}$.

- Cas $L=\varnothing$. Notons $U_{1}=V_{3}, U_{2}=V_{2}, C=S \cup \gamma=S \cup \widehat{\gamma}, A=C \cap \bar{U}_{2}$, $B=C \backslash U_{1}$ et $\widetilde{A}=A \cap \partial U_{2}=\ell$. De plus, comme $B \subset S$, en appliquant le théorème de Siu [36] à $S$ dans des voisinages de $B$, on obtient que $B$ admet une base de voisinages de Stein. De plus, comme $L=\varnothing, \ell$ est holomorphiquement convexe dans $V$ et donc il admet une base de voisinages $\left(W_{i}\right)$ dont l'adhérence est holomorphiquement convexe dans $V$. D'après [8], [9], $\left({\overline{W_{i} \cup \gamma}}\right) \backslash\left(\bar{W}_{i} \cup \gamma\right)$ est un sous-ensemble analytique $R_{i}$ de dimension pure 1 et de masse finie de $V \backslash\left(\bar{V}_{\tilde{A}} \cup \gamma\right)$ contenant $\widehat{\gamma} \backslash \gamma$.

LEMME 5.13. - Il existe un ouvert $W_{i_{0}}$ tel que $\left(\widehat{\bar{W}_{i_{0}} \cup \gamma}\right) \backslash\left(\overline{W_{i_{0}}} \cup \gamma\right) \subset S$.

TOME $130-2002-\mathrm{N}^{\mathrm{O}} 2$ 
Démonstration. - Soit $W_{i}$ un voisinage de $\ell$ dont l'adhérence est holomorphiquement convexe dans $V$ et notons

$$
R_{i}=\left(\widehat{\overline{W_{i}} \cup \gamma}\right) \backslash\left(\overline{W_{i}} \cup \gamma\right) \subset S .
$$

Alors $R_{i}$ est un sous-ensemble analytique de dimension pure 1 et de masse finie de $V \backslash\left(\overline{W_{i}} \cup \gamma\right)$. Comme $\bar{W}_{i}$ est holomorphiquement convexe dans $V$, d'après le principe du maximum, il n'existe pas de sous-ensemble analytique de dimension pure 1 de $V \backslash \overline{W_{i}}$ borné dans $V$. Par conséquent, $R_{i}$ ne contient qu'un nombre fini de composantes analytiques irréductibles $R_{1}, \ldots, R_{k_{i}}$ dont le bord intersecte $\bar{W}_{i}$. Comme $\widehat{\gamma} \backslash \gamma \subset S$ par construction, toute composante irréductible de $R_{i}$ non incluse dans $S$ est égale à l'une des $R_{1}, \ldots, R_{k_{i}}$. De plus, par définition de l'enveloppe holomorphiquement convexe, si $W_{j} \subset W_{i}$ alors

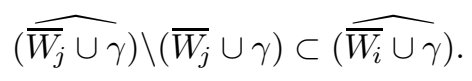

Par conséquent, $R_{j} \backslash\left(\overline{W_{i}} \cup \gamma\right)$ est inclus dans $R_{i}$. Si pour tout $j \in J$ tel que $W_{j} \subset \subset W_{i}$, il existe une composante irréductible $D$ de $R_{j}$ non incluse dans $S$, alors $D \backslash \overline{W_{i}}$ est l'une des composantes $R_{1}, \ldots, R_{k_{i}}$. Mais alors, il existerait une suite croissante d'ensembles analytiques irréductibles dont la limite serait un sous-ensemble analytique $D_{\infty}$ de $V \backslash(\gamma \cup \ell)$ borné dans $V$. On a $D_{\infty} \subset$ $\widehat{(\ell \cup \gamma)} \backslash(\ell \cup \gamma) \subset S$, ce qui donne la contradiction recherchée.

Et donc en posant $V_{\tilde{A}}=W_{i_{0}}$, l'ensemble $\bar{V}_{\tilde{A}} \cup A$ est holomorphiquement convexe dans $V$ et admet donc une base de voisinages de Stein. D'après le lemme 5.8, $C$ admet une base de voisinages de Stein ce qui termine la preuve de la proposition 5.8 dans le premier cas.

Cas général. - Notons $K=L \cap \gamma$ alors par construction de $S$ et $\gamma$ et d'après le théorème d'unicité [8], $K$ est un compact de longueur nulle de $V$. De plus, quitte à ajouter un nombre fini de points à $K$, on peut toujours supposer que $K$ intersecte toutes les composantes irréductibles de $L$.

LEMME 5.14. - Il existe une base de voisinages de $K \cup \widehat{\alpha}$ par des ouverts $W_{i}$ vérifiant les propriétés suivantes :

1) leur adhérence est holomorphiquement convexe dans $V$;

2) leur bord est transverse à $S$, $\gamma$ et $L$;

3) $r_{i}:=L \cap \partial W_{i}$ est une courbe lisse tel qu'il n'existe pas de composante irréductible de $S \backslash W_{i}$ dont le bord est inclus dans $r_{i}$.

Démonstration. - Comme $K$ est de longueur nulle, $K \cup \widehat{\alpha}$ est holomorphiquement convexe dans $V$. Par conséquent, il admet une base de voisinage par des ouverts dont l'adhérence est holomorphiquement convexe dans $V$. Bien sûr, on peut toujours supposer que ces ouverts ont un bord lisse transverse à $S$ et $\gamma$. Enfin si pour chaque ouvert $W_{i}$, il existe une composante de $S \backslash W_{i}$ dont le 
bord est inclus dans $r_{i}$, nous obtenons une suite croissante d'ensembles analytiques $R_{i}$ inclus dans $S \backslash W_{i}$ dont le bord est entièrement inclus dans $\bar{W}_{i}$. Mais alors la réunion $R_{\infty}$ des $R_{i}$ est un sous-ensemble analytique de $S \backslash K$ dont le bord est entièrement inclus dans $K$. Puisque $K$ est de longueur nulle et $S$ est de masse finie, d'après [5], [26], [20], [35], [2], $\bar{R}$ est une courbe compacte de $X$ incluse dans $S \cup \gamma$, ce qui donne la contradiction recherchée.

Soient $W_{j+1} \subset \subset W_{j}$ deux ouverts obtenus grâce au lemme précédent. Posons

$$
S_{2}=S \backslash \overline{W_{j+1}} \quad \text { et } \quad \gamma_{2}=\left(\gamma \backslash W_{j+1}\right) \cup \partial S_{2} .
$$

LEMME 5.15. - Le sous-ensemble analytique $\widehat{\gamma}_{2} \backslash \gamma_{2}$ admet un nombre fini de composantes irréductibles.

Démonstration. - On a $\gamma \backslash W_{j+1} \subset \widetilde{\gamma} \subset \bar{S}_{2}$. Donc $\gamma_{2}$ est contenu dans $\bar{S}_{2}$ et on a en fait $\gamma_{2} \subset \partial\left(S \backslash W_{j+1}\right)$. Par construction $W_{j+1} \supset K \cup \widehat{\alpha} \supset \overline{(S \backslash \widetilde{S})}$. Par conséquent $\gamma_{2} \subset \widetilde{\widetilde{S} \backslash W_{j+1}} \subset L$. L'hypothèse de transwersalite du bord de $W_{j+1}$ permet alors de conclure.

Soit $H$ une hypersurface complexe de $V$ évitant $\gamma_{2} \cup S_{2}$ et intersectant dans $W_{j+1}$ toutes les composantes de $L$ et de $\widehat{\gamma}_{2} \backslash \gamma_{2}$. Alors $V \backslash H$ est un ouvert de Stein et $\ell=S_{2} \cap \partial V_{2}$ est holomorphiquement convexe dans $V \backslash H$. Par conséquent, d'après le premier cas, $\gamma_{2} \cup S_{2}$ admet une base de voisinage de Stein. Notons alors $C=S \cup \gamma, U_{1}=X \backslash W_{j+1}, U_{2}=X \backslash W_{j}, A=C \cap \bar{U}_{2}, B=C \backslash U_{1}$ et $\widetilde{A}=A \cap \partial U_{2}$. Alors $B$ admet une base de voisinage de Stein car $S \cup \gamma \cap \bar{W}_{j}$ est holomorphiquement convexe dans $V$. Soit $Z$ un voisinage de Stein de $A$, alors d'après le lemme 5.14, $\widetilde{A}$ est holomorphiquement convexe dans $Z$ et admet donc un voisinage $V_{\tilde{A}}$ dont l'adhérence est holomorphiquement convexe dans $Z$. Par conséquent $\bar{V}_{\tilde{A}} \cup A$ admet une base de voisinage de Stein. Le lemme 5.9 montre alors que $C$ admet un voisinage de Stein, ce qui termine la preuve de la proposition.

\subsection{Existence d'une solution au problème du bord de support minimal}

Proposition 5.16. - Soient $X$ une variété complexe disque-convexe (munie d'une métrique complète) et $[\gamma]$ un 1-courant rectifiable fermé dont le support $\gamma$ est un compact de classe $A_{1}$. Soit $\mathcal{S}$ l'ensemble des solutions au problème $d u$ bord pour $[\gamma]$ (i.e. l'ensemble des 1-chaînes holomorphes $[S]$ de masse finie de $X \backslash \gamma$ dont le support est relativement compact dans $X$ et telle que $d[S]=[\gamma]$ ). Supposons que $\mathcal{S}$ n'est pas vide, alors il existe une 1-chaîne holomorphe $\left[S_{\min }\right]$ solution au problème du bord pour $[\gamma]$ dont le volume du support est égal à

$$
I=\inf _{[S] \in \mathcal{S}} \operatorname{Vol} S
$$

où $S$ est le support de la 1-chaîne holomorphe $[S]$.

TOME $130-2002-\mathrm{N}^{\mathrm{O}} 2$ 
Démonstration. - Soit $[S]=\sum_{i \in J} m_{i}\left[S_{i}\right]$ une solution au problème du bord pour $[\gamma]$ où les $S_{i}$ sont les composantes irréductibles de $S \backslash \gamma$. Soit $V$ un voisinage de Stein de $\gamma$ et pour tout $\nu$, soit $W_{\nu} \subset V$ un $\nu$-voisinage de $\gamma$, posons

$$
\left[S^{\nu}\right]=\sum_{\substack{i \in J \\ S_{i} \subset W_{\nu}}} m_{i}\left[S_{i}\right] .
$$

Soit $\nu$ tel que

$$
\operatorname{Vol} S^{\nu}<\frac{1}{4} C
$$

où $C$ est une constante (obtenue grâce à la proposition 5.7) telle que tout sousensemble analytique irréductible de $X \backslash \gamma$ donc l'adhérence intersecte $\gamma$ et $X \backslash V$ ait un volume strictement supérieur à $C$. $S$ étant de volume fini, il existe alors un nombre fini de composantes irréductibles $\left\{S_{i}^{\nu}\right\}_{i=1 . . N}$ de $S$ non incluses dans $W_{\nu}$. Pour toute composante irréductible $S_{i}^{\nu}$ notons, si elle existe, $\widetilde{S}_{i}^{\nu}$ une surface de Riemann compacte irréductible de $X$ contenant $S_{i}^{\nu}$ et $\widetilde{S}_{i}^{\nu}=\varnothing$ sinon. Posons

$$
A=S \cup\left(\bigcup_{i=1, \ldots, N} \widetilde{S}_{i}^{\nu}\right)
$$

et montrons que le support de toute solution au problème du bord $[R]$ pour $[\gamma]$ vérifiant $\operatorname{Vol} R \leq I+\frac{1}{2} C$ est inclus dans $A$. On a

$$
[R]-[S]=\sum_{i=1}^{M} n_{i}\left[V_{i}\right]
$$

où les $\left[V_{i}\right]$ sont les courants d'intégration sur des surfaces de Riemann compactes irréductibles $V_{i}$. Si $V_{i}$ contient une composante irréductible $S_{j}^{\nu}$ de $S \backslash \gamma$ non incluse dans $W_{\nu}$, on a $V_{i} \subset \widetilde{S}_{j}^{\nu} \subset A$ par définition de $\widetilde{S}_{j}^{\nu}$. Donc, si pour tout $i \in\{1, \ldots, M\}, V_{i}$ contient une composante irréductible $S_{j}^{\nu}$ de $S \backslash \gamma_{x}$ non incluse dans $W_{\nu}$, on a $\operatorname{supp}([R]-[S]) \subset A$ et donc $R \subset A$. Dans le cas contraire, il existe un $i_{0} \in\{1, \ldots, M\}$ tel que $V_{i_{0}}$ ne contient que des composantes irréductibles de $S \backslash \gamma_{x}$ incluse dans $W_{\nu}$. Soit $[\widetilde{R}]$ (resp. $[\widetilde{S}]$ ) la restriction de $[R]$ (resp. de $[S]$ ) à $V_{i_{0}}$. On a alors par construction

$$
\operatorname{Vol} \widetilde{S} \leq \operatorname{Vol} S^{\nu}<\frac{1}{4} C .
$$

Or $[\widetilde{R}]=[\widetilde{S}]+n_{i_{0}}\left[V_{i_{0}}\right]\left(\right.$ avec $n_{i} \neq 0$ ), et donc, comme $\widetilde{S} \subset V_{i_{0}}$ et que $V_{i_{0}}$ est de volume strictement supérieur à $C$ car, étant compact, il ne peut être inclus dans $V$, on a :

$$
\operatorname{Vol} \widetilde{R} \geq \operatorname{Vol} V_{i_{0}}-\operatorname{Vol} \widetilde{S}>\frac{3}{4} C .
$$

Le courant $[R]-[\widetilde{R}]+[\widetilde{S}]$ est encore solution au problème du bord pour $[\gamma]$. De plus, $\operatorname{supp}([R]-[\widetilde{R}])$ et $\widetilde{S}$ n'ont pas de composantes analytiques communes. 
En effet, par construction, $\operatorname{supp}([R]-[\widetilde{R}])$ n'a pas de composante analytique incluse dans $V_{i_{0}}$ et $\widetilde{S} \subset V_{i_{0}}$. Par conséquent on a

$$
\operatorname{Vol}(\operatorname{supp}([R]-[\widetilde{R}]+[\widetilde{S}]))=\operatorname{Vol}(\operatorname{supp}([R]-[\widetilde{R}]))+\operatorname{Vol}(\widetilde{S}) .
$$

De même, $[R]=([R]-[\widetilde{R}])+[\widetilde{R}]$ et $\operatorname{supp}([R]-[\widetilde{R}])$ et $\widetilde{R}$ sont par construction sans composantes analytiques communes et donc

$$
\operatorname{Vol}(R)=\operatorname{Vol}(\operatorname{supp}([R]-[\widetilde{R}]))+\operatorname{Vol}(\widetilde{R}) .
$$

Par conséquent

$$
\begin{aligned}
\operatorname{Vol}(\operatorname{supp}([R]-[\widetilde{R}]+[\widetilde{S}])) & =\operatorname{Vol}(\operatorname{supp}([R]-[\widetilde{R}]))+\operatorname{Vol} \widetilde{S} \\
& =\operatorname{Vol}(R)-\operatorname{Vol}(\widetilde{R})+\operatorname{Vol}(\widetilde{S}) \\
& <\left(I+\frac{C}{2}\right)-\frac{3 C}{4}+\frac{C}{4} \leq I
\end{aligned}
$$

ce qui donne la contradiction recherchée et prouve que $R \subset A$. L'ensemble $A$ ne contenant qu'un ensemble fini de surfaces de Riemann compactes, on est réduit au cas où $\gamma$ est inclus dans une surface de Riemann irréductible $L$. Si $\left[R_{1}\right]$ et $\left[R_{2}\right]$ sont deux solutions distinctes au problème du bord pour $[\gamma]$ dans $L$, on a $\left[R_{1}\right]-\left[R_{2}\right]=m[L]$ pour un certain $m \in \mathbb{Z}$. En particulier, si deux solutions sont nulles sur une composante irréductible de $L \backslash \gamma$, elles sont égales. Il existe donc une solution au problème du bord pour $[\gamma]$ dont le complémentaire du support est de volume maximal et donc tel que le support est de volume minimal.

\subsection{Existence d'une solution globale}

Proposition 5.17. - Sous les hypothèses du théorème 2.3, supposons que la proposition 3 de ce théorème est vérifiée, que $\Gamma$ est connexe et de dimension 3 et qu'il existe une solution au problème du bord $[T]$ pour $[\Gamma]$ dans $(U \backslash F) \times \omega$. Alors il existe une 2-chaîne holomorphe $[\widetilde{T}]$ de $U \times \omega \backslash \Gamma$, solution au problème $d u$ bord pour $[\Gamma]$ dans $U \times \Omega$ et qui coïncide avec $[T]$ dans $(U \backslash F) \times \omega$.

Démonstration. - Nous dirons que $\Gamma$ admet une extension analytique au point $x \in \Gamma$, s'il existe un voisinage $V_{x}$ de $x$ et un sous-ensemble analytique $T_{x}$ de $V_{x} \backslash \Gamma$, de volume fini et tel que $[\Gamma]= \pm d\left[T_{x}\right]$ dans $V_{x}$. D'après le théorème de Trépreau [38], si $\Gamma$ est minimale au point $x$, elle admet une extension analytique au point $x$. De plus, si $\gamma:[0,1] \rightarrow \Gamma$ est une courbe CR et si $\Gamma$ admet une extension analytique au voisinage de $\gamma(0)$ alors elle admet une extension analytique au voisinage de $\gamma(1)$.

LEMME 5.18. - $\Gamma$ admet une extension analytique en chacun de ses points.

Démonstration. - Par hypothèse, $\Gamma$ admet une extension analytique en tout point de $\Gamma \cap \pi^{-1}(U \backslash F)$. Soit $x \in \Gamma \cap \pi^{-1}(F)$, il faut montrer que $\Gamma$ admet une extension analytique en $x$. Soit $\mathcal{O}_{C R}(x)$ l'orbite $\mathrm{CR}$ de $x$ dans $\Gamma$. Si cette orbite est un ouvert de $\Gamma$ alors $\Gamma$ est minimal au point $x$ est admet donc une extension

TOME $130-2002-\mathrm{N}^{\mathrm{O}} 2$ 
analytique. Sinon, $\mathcal{O}_{\mathrm{CR}}(x)$ contient une surface de Riemann lisse $H_{x} \ni x(M$ est supposé de dimension 3). Or d'après les propriétés $b$ et $c, H_{x} \not \subset \pi^{-1}(F)$ et il existe donc un point $y \in H_{x}$ tel que $\Gamma$ admette une extension analytique au point $y$. Du fait de la propriété d'extension analytique le long des courbes CR, on a montré que $\Gamma$ admet une extension analytique au point $y$.

Quitte à déformer légèrement $M$ dans l'extension analytique à un côté obtenu dans le lemme précédent, on peut donc supposer qu'il existe un voisinage ouvert $W$ de $\Gamma$ et un sous-ensemble analytique irréductible $\widetilde{T}$ de $W \backslash \Gamma$ extension analytique de $T$ (i.e. tel que $d[\widetilde{T}]= \pm[\Gamma]$ et $\widetilde{T} \cap \pi^{-1}(U \backslash F) \cap W=T \cap W$. Par conséquent, quitte à encore déformer $\Gamma$, on peut supposer que l'ensemble $K$ définit dans le lemme 2.7 est vide. Par conséquent, la preuve du théorème principal donne alors que l'ensemble $F$ n'est pas 1-générique. C'est-à-dire que $F$ est inclus dans un ensemble dénombrable de points. Or $F$ est fermé par construction. Le théorème du type Thullen de Siu permet alors de montrer que $F$ n'admet pas de points isolés et donc que $F$ est vide.

\section{BIBLIOGRAPHIE}

[1] Alexander (H.) - Polynomial approximation and hulls in sets of finite linear measure in $\mathbb{C}^{n}$, Amer. J. Math., t. 93 (1971), pp. 65-74.

[2] Holomorphic chains and the support hypothesis conjecture, J. Amer. Math. Soc., t. 10 (1997), pp. 123-138.

[3] Andreotti (A.) \& Siu (Y.) - Projective embedding of pseudoconcave space, Ann. Scuola Norm. Sup. Pisa, t. 24 (1970), no. 3, pp. 231-278.

[4] Barrett (D.E.) - A remark on the global embedding problem for threedimensional CR manifolds, Proc. Amer. Math. Soc., t. 102 (1988), no. 4, pp. 888-892.

[5] Bishop (E.) - Condition for the analyticity of certain sets, Michigan Math. J., t. 482 (1964), pp. 289-304.

[6] Boutet De Monvel (L.) - Intégration des équations de CauchyRiemann induites formelles, exposé IX, Séminaire Goulaouic-LionsSchwartz, 1974-1975.

[7] Chirka (E.M.) - Complex Analytic Sets, Kluwer Academic Publishers Group, Dordrecht, 1989.

[8] Dinh (T.C.) - Enveloppe polynomiale d'un compact de longueur finie et chaînes holomorphes à bord rectifiable, Acta Math., t. 180 (1998), no. 1, pp. 31-67.

[9] _ Orthogonal measures on the boundary of a Riemann surface and polynomial hull of compacts of finite length, J. Funct. Anal., t. 157 (1998), pp. 624-649.

BULLETIN DE LA SOCIÉtÉ MATHÉMATIQUE DE FRANCE 
[10] _ Problème du bord dans l'espace projectif complexe, Ann. Inst. Fourier, t. 45 (1998), no. 5, pp. 1483-1512.

[11] Sur la caractérisation du bord d'une variété complexe dans l'espace projectif, Bull. Soc. Math. France, t. 127 (1999), pp. 519-539.

[12] Dolbeault (P.) \& Henkin (G.) - Chaînes holomorphes de bord donné dans un ouvert $q$-concave de $\mathbb{C} P^{n}$, Bull. Soc. Math. France, t. 125 (1997), pp. 383-445.

[13] FAlBel (E.) - Non-embeddable CR-manifolds and surface singularities, Invent. Math., t. 108 (1992), pp. 49-65.

[14] Federer (H.) - Geometric Measure Theory, Grundlehren Math. Wiss., vol. 153, Springer-Verlag, New York, 1969.

[15] Grauert (H.) - On the Levi's problem and the embedding of real-analytic manifolds, Ann. of Math., t. 68 (1958), pp. 460-472.

[16] Sheaf-theorical methods in complex analysis, Several complex variables VII, Springer-Verlag, Berlin, 1994.

[17] Harvey (R.) - Holomorphic chains and their boundaries, in Several complex variables (Proc. Sympos. Pure Math., Vol. XXX, Part 1, Williams Coll., Williamstown, Mass., 1975), Amer. Math. Soc., 1977, pp. 309-382.

[18] Harvey (R.) \& LAWson (B.) - On boundaries of complex analytic varieties, I, Ann. of Math., t. 102 (1975), pp. 233-290.

[19] _ On boundaries of complex analytic varieties, II, Ann. of Math., t. 106 (1977), pp. 213-238.

[20] Harvey (R.) \& Shiffman (B.) - A characterization of holomorphic chains, Ann. of Math., t. 99 (1974), no. 2, pp. 553-587.

[21] Henkin (G.) - H. Lewy's equation and analysis on pseudoconvex manifolds, Uspehi. Mat. Nauk., t. 32 (1977), no. 3 (195), pp. 57-118, 247.

[22] Ivashkovich (S.M.) - The Hartogs-type extension theorem for meromorphic maps into compact Kähler manifolds, Invent. Math., t. 109 (1992), pp. $47-54$.

[23] Complex Plateau problem in non Kähler manifolds, Ann. Polon. Math., t. 70 (1998), pp. 131-143.

[24] JÖRICKE (B.) - Some remarks concerning holomorphically convexe hulls and envelopes of holomorphy, Math. Z., t. 218 (1995), pp. 143-157.

[25] Kato (M.) - Compact complex surfaces containing global strongly pseudoconvex hypersurfaces, Tôhoku Math. J., t. 31 (1979), pp. 537-547.

[26] King (J.) - The currents defined by analytic varieties, Acta Math., t. 127 (1971), pp. $185-220$.

[27] Konn (J.J.) - Several complex variables from the point of view of linear partial differential equations, Proc. Sympos. Pure Math., t. XXX, Part 1 (1975), pp. 215-237.

[28] Lawrence (M.G.) - Polynomial hulls of sets of finite length in strictly convexe boundaries, manuscript.

TOME $130-2002-\mathrm{N}^{\mathrm{O}} 2$ 
[29] Mihalache (N.) - Voisinages de Stein pour les surfaces de Riemann avec bord immergées dans l'espace projectif, Bull. Sci. Math., t. 120 (1996), no. 4, pp. 397-404.

[30] Porten (E.) - A Hartogs-Bochner type theorem for continuous CRmappings, manuscript, 1996.

[31] Rossi (H.) - Attaching analytic spaces to an analytic space along a pseudoconcave boundary, in Proc. Conf. Complex Analysis, Springer, Berlin, 1965, pp. 242-256.

[32] _ Homogeneous strongly pseudoconvex hypersurfaces, Rice Univ. Studies, t. 59 (1973), no. 1, pp. 131-145.

[33] SARKIS (F.) - CR-meromorphic extension and the non-embedding of the Andreoti-Rossi CR-structure in the projective space, Int. J. Math., t. 10 (1999), pp. 897-915.

[34] _ Problème du bord dans es variétés kählériennes et extension des fonctions CR-méromorphes, Thèse, Université Paris VI, 1999.

[35] Shiffman (B.) - Complete characterization of holomorphic chains of codimension one, Math. Ann., t. 274 (1986), pp. 233-256.

[36] SIu (Y.) - Every Stein Subvariety Admits a Stein Neighborhood, Invent. Math., t. 38 (1976), pp. 89-100.

[37] Stolzenberg (G.) - Uniform approximation on smooth curves, Acta Math., t. 115 (1966), pp. 185-198.

[38] TrÉpreau (J.M.) - Sur la propagation des singularités dans les variétés $C R$, Bull. Soc. Math. France, t. 118 (1990), pp. 403-450.

[39] Wermer (J.) - The hull of a curve in $\mathbb{C}^{n}$, Ann. of Math., t. 68 (1958), pp. 550-561. 